\title{
The COS Absorption Survey of Baryon Harbors: The Galaxy Database and Cross- correlation Analysis of O VI Systems*
}

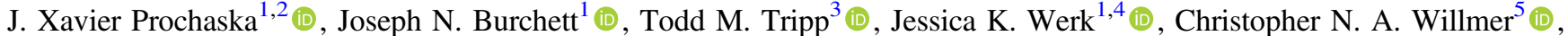 \\ J. Christopher Howk ${ }^{6}(1)$, Scott Lange ${ }^{1}$, Nicolas Tejos ${ }^{7}$, Joseph D. Meiring ${ }^{3}$, Jason Tumlinson ${ }^{8,9}$ (i), Nicolas Lehner ${ }^{6}$ (iD, \\ Amanda B. Ford ${ }^{10}$, and Romeel Davé ${ }^{11}$ \\ ${ }^{1}$ University of California, Santa Cruz; 1156 High St., Santa Cruz, CA 95064, USA; xavier@ucolick.org \\ ${ }^{2}$ Kavli Institute for the Physics and Mathematics of the Universe (Kavli IPMU) The University of Tokyo; 5-1-5 Kashiwanoha, Kashiwa, 277-8583, Japan \\ ${ }^{3}$ Department of Astronomy, University of Massachusetts, 710 North Pleasant Street, Amherst, MA 01003-9305, USA \\ ${ }^{4}$ University of Washington, Department of Astronomy, Seattle, WA 98195, USA \\ ${ }^{5}$ Steward Observatory, University of Arizona, 933 North Cherry Avenue, Tucson, AZ 85721, USA \\ ${ }^{6}$ Department of Physics, University of Notre Dame, 225 Nieuwland Science Hall, Notre Dame, IN 46556, USA \\ ${ }^{7}$ Instituto de Física, Pontificia Universidad Católica de Valparaíso, Casilla 4059, Valparaíso, Chile \\ ${ }^{8}$ Space Telescope Science Institute, Baltimore, MD 21218, USA \\ ${ }^{9}$ Department of Physics and Astronomy, Johns Hopkins University, Baltimore, MD 21218, USA \\ ${ }^{10}$ Google, 1600 Amphitheatre Parkway, Mountain View, CA 94043, USA \\ ${ }^{11}$ Institute for Astronomy, Royal Observatory, University of Edinburgh, EH9 3HJ, UK \\ Received 2018 September 6; revised 2019 June 17; accepted 2019 June 19; published 2019 July 30
}

\begin{abstract}
We describe the survey for galaxies in the fields surrounding nine sightlines to far-UV bright, $z \sim 1$ quasars that define the COS Absorption Survey of Baryon Harbors (CASBaH) program. The photometry and spectroscopy that comprise the data set come from a mixture of public surveys (SDSS, DECaLS) and our dedicated efforts on private facilities (Keck, MMT, LBT). We report the redshifts and stellar masses for 5902 galaxies within $\approx 10$ comovingMpc of the sightlines with a median of $\bar{z}=0.28$ and $\bar{M}_{*} \approx 10^{10.1} M_{\odot}$. This data set, publicly available as the CASBaH SPECDB, forms the basis of several recent and ongoing CASBaH analyses. Here, we perform a clustering analysis of the galaxy sample with itself (auto-correlation) and against the set of O VI absorption systems (crosscorrelation) discovered in the CASBaH quasar spectra with column densities $N\left(\mathrm{O}^{+5}\right) \geqslant 10^{13.5} \mathrm{~cm}^{-2}$. For each, we describe the measured clustering signal with a power-law correlation function $\xi(r)=\left(r / r_{0}\right)^{-\gamma}$ and find that $\left(r_{0}, \gamma\right)=\left(5.48 \pm 0.07 h_{100}^{-1} \mathrm{Mpc}, 1.33 \pm 0.04\right)$ for the auto-correlation and $\left(6.00_{-0.77}^{+1.09} h_{100}^{-1} \mathrm{Mpc}, 1.25 \pm 0.18\right)$ for galaxy-O VI cross-correlation. We further estimate a bias factor of $b_{\mathrm{gg}}=1.3 \pm 0.1$ from the galaxy-galaxy autocorrelation, indicating the galaxies are hosted by halos with mass $M_{\text {halo }} \approx 10^{12.1 \pm 0.05} M_{\odot}$. Finally, we estimate an $\mathrm{O}$ VI-galaxy bias factor $b_{\mathrm{O} \text { VI }}=1.0 \pm 0.1$ from the cross-correlation which is consistent with $\mathrm{O}$ VI absorbers being hosted by dark matter halos with typical mass $M_{\text {halo }} \approx 10^{11} M_{\odot}$. Future works with upcoming data sets (e.g., $\mathrm{CGM}^{2}$ ) will improve upon these results and will assess whether any of the detected $\mathrm{O}$ VI arises in the intergalactic medium.
\end{abstract}

Key words: astronomical databases: miscellaneous - galaxies: evolution - intergalactic medium - quasars: absorption lines

Supporting material: machine-readable table

\section{Introduction}

The cosmic web is the filamentary network of dark matter and baryons predicted by cosmological simulations to permeate our universe (Miralda-Escudé et al. 1996; Lukić et al. 2015). It forms under the competing influences of gravitational collapse and cosmic expansion, modulated by hydrodynamic heating and cooling during collapse, and also ionization balance with the extragalactic radiation field. While the web holds as a ubiquitous prediction of dark matter cosmology, tests of this paradigm are relatively scarce.

Using luminous galaxies as tracers, wide-field surveys have revealed patterns of large-scale structure that resemble theoretical prediction. And, within the limited statistical measures

\footnotetext{
* Based on observations made with the NASA/ESA Hubble Space Telescope, obtained at the Space Telescope Science Institute, which is operated by the Association of Universities for Research in Astronomy, Inc., under NASA contract NAS 5-26555. These observations are associated with programs 13033 and 11598. Partly based on observations taken at the MMT Observatory, a joint facility operated by the Smithsonian Institution and the University of Arizona.
}

afforded by these data, the distributions match model predictions (e.g., Davis et al. 1985; Bond et al. 1996). As the statistical sample increases and pushes to higher redshift, topology diagnostics afford tests of the cosmic web morphology (e.g., Cautun et al. 2013; Tempel et al. 2014). These experiments, however, are inherently limited by the sparseness of galaxies and the surveys' inherent biases (e.g., Smith et al. 2003).

Lacking a means to directly image the diffuse emission predicted from the cosmic web (Gould \& Weinberg 1996; Cantalupo et al. 2014), one relies on the inverse approach of detecting the web's threads in absorption. Absorption lines in the spectra of luminous background sources (typically quasars) yield a one-dimensional description of the matter distribution across cosmic time. Quantitative comparison of cosmological predictions with the resultant $\mathrm{HI}$ Ly $\alpha$ forest have lent further support to this model (e.g., Miralda-Escudé et al. 1996; Croft et al. 2002). The agreement is sufficiently compelling that modern efforts have inverted the practice, adopting the cosmic 
web paradigm to constrain parameters of the cosmology and other properties of the universe (e.g., Palanque-Delabrouille et al. 2013; Slosar et al. 2013).

Within these same quasar spectra, one also identifies absorption from transitions of heavy elements (e.g., C IV, $\mathrm{O}$ VI) that record prior enrichment by galaxies. The high incidence of this metal absorption, especially at high- $z$ where the data quality is exquisite and the rest-frame ultraviolet (UV) transitions shift into the optical bandpass and become observable from ground based telescopes, requires this enrichment to extend far beyond the galaxies' interstellar medium (ISM) and possibly beyond their local environs (the circumgalactic medium or CGM) and into the IGM (e.g., Simcoe et al. 2004; Booth et al. 2012). The distribution of enrichment on these scales is a complex interplay between the timing of the metal production, the galaxies involved, the processes that eject/transport the matter from star-forming regions, and the underlying potential well of the galaxy and its environment. Unfortunately, the degree of complexity is such that even a precise accounting of the incidence and degree of heavy element absorption along multiple sightlines is insufficient to fully resolve the underlying astrophysics (e.g., Ford et al. 2016).

Of greater potential power for analyzing the cosmic web and its enrichment is to combine absorption-line studies with surveys of the galaxies surrounding the absorption-line sightlines. At $z \sim 0$, where galaxies are more easily observed, several studies have examined UV spectroscopy of the Ly $\alpha$ forest to provide constraints on the present-day cosmic web (Morris et al. 1993; Bowen et al. 2002; Penton et al. 2002; Chen et al. 2005; Aracil et al. 2006; Wakker \& Savage 2009; Prochaska et al. 2011b; Tejos et al. 2012). These have established the connection between intergalactic filaments and Ly $\alpha$ absorption (Wakker et al. 2015) and also glimpses of the voids which are expected to fill the volume (Tejos et al. 2012). Studies focused on the association of heavy elements to the cosmic web are more rare (Aracil et al. 2006; Stocke et al. 2006; Chen \& Mulchaey 2009; Prochaska et al. 2011b) and these are stymied by smaller samples. Prochaska et al. (2011b) argued that the majority (and possibly all) of the metal-line detections in quasar spectra arise within a few hundred kpc of galaxies, casting some doubt for any enrichment in the IGM. These results, however, were tempered by the limitations of sample variance and the signal-to-noise and detection sensitivity of the UV absorption spectra.

Extending absorber-galaxy analysis to $z>0$ is challenged by several evolving factors. For $z \sim 0-1$, the paucity of UVbright quasars limits the number and quality of absorption-line spectra available. Furthermore, current wide-field surveys (SDSS, 2dF) are generally complete only at $z<0.1$. Primary exceptions are those targeting large samples of luminous red galaxies (LRGs; Eisenstein et al. 2001) and, more recently, emission line galaxies (Dawson et al. 2013). While these $z \sim 0.5$ galaxy surveys have enabled important works on a subset of absorption lines (primarily Mg II; Zhu et al. 2014; Lan \& Mo 2018), detailed exploration of the cosmic web has required dedicated follow-up surveys of the rare fields hosting UV-luminous quasars (Prochaska et al. 2011a; Tejos et al. 2014; Johnson et al. 2015; Keeney et al. 2018).

The most comprehensive work at $z \sim 0.5$ has been carried out by a group in Durham with results published by Tejos et al. (2014) and Finn et al. (2016). The first paper focused on H I absorption and its clustering to galaxies on scales of $\sim 10 h_{100}^{-1} \mathrm{Mpc}$. Their analysis confirmed previous assertions (Morris et al. 1993; Tripp et al. 1998; Chen et al. 2005) that the $\mathrm{H} \mathrm{I} \mathrm{Ly} \alpha$ forest is roughly divided into a low-density population tracing the cosmic web and a higher-density component associated with dark matter halos. The second paper measured the cross-correlation of $\mathrm{O}$ VI with galaxies which they interpreted as evidence for $\mathrm{O}$ VI distributed away from galaxies but following the same underlying mass distribution on $\sim \mathrm{Mpc}$ scales.

With the installation of the Cosmic Origins Spectrograph (COS) on the HST in 2009, we were motivated to pursue a new survey dedicated to investigations of the cosmic web at $z \sim 0-1.5$. With this goal in mind, the COS Absorption Survey of Baryon Harbors (CASBaH; HST Programs $11741 \& 13846$, PI Tripp) was initiated to obtain high signal-to-noise ratio $(\mathrm{S} / \mathrm{N})$ spectra of $\sim 10$ quasars at $z \gtrsim 1$ and to assess $\mathrm{HI}$ and heavy element absorption. A full description of the CASBaH design and data handling procedures, as well as the first release of the absorption-line database, are provided by T. Tripp et al. (2019, in preparation). In brief, this survey observed nine quasi-stellar objects (QSOs) (see Table 1) with the high-resolution COS G130M, G160M, G185M, and G225M gratings as well as the Space Telescope Imaging Spectrograph (STIS) E230M echelle mode. ${ }^{12}$ This set of observations was designed to provide complete spectral coverage from observed wavelength $\lambda_{\mathrm{ob}}=1152 \AA$ to $\left(1+z_{\mathrm{QSO}}\right) \times 1215.67 \AA$, i.e., the spectra cover the entire Ly $\alpha$ forest for each QSO with good spectral resolution $\left(\mathrm{FWHM} \approx 10-20 \mathrm{~km} \mathrm{~s}^{-1}\right)$. In the far-UV (FUV) range $\left(\lambda_{\mathrm{ob}}=1152-1800 \AA\right)$, the survey was designed to detect weak metal lines such as the Ne VIII doublet and affiliated species (e.g., Tripp et al. 2011; Meiring et al. 2013), and accordingly the exposure times were set to provide $S /$ Ns of $\approx 15-50$ per resolution element. The near-UV (NUV) spectra were obtained to detect strong $\mathrm{H}$ I lines that are crucial for proper line/system identification and to extend the coverage of strong $\mathrm{O}$ VI lines to higher redshifts; for these purposes, the NUV data did not require high $\mathrm{S} / \mathrm{N}$ and typically had $\mathrm{S} / \mathrm{N} \approx 5-20$ per resolution element. The achieved S/N levels of the FUV spectra (the COS G130M and G160M data) afford unparalleled insight into the diffuse gas of the cosmic web and access to extreme UV lines (e.g., Ne VIII) that have been only rarely observed.

A crucial component of the CASBaH program is a dedicated, deep survey of galaxies around the quasar sightlines. This paper describes the $\mathrm{CASBaH}$ galaxy redshift survey and provides the current CASBaH galaxy database. The presentation of this database culminates many years of observing to gather $\sim 10,000$ spectra in seven quasar fields. Given the tremendous legacy value of the $\mathrm{CASBaH}$ absorption-line database, there are certain to be additional surveys of galaxies in these fields (e.g., QSAGE; R. Bielby et al. 2019, in preparation). This paper additionally offers a first analysis of the nature of $\mathrm{OVI}$ absorption in the cosmic web. Other upcoming works from CASBaH include detailed analyses of the gas ionization state and absorption kinematic structure that leverage the very high $\mathrm{S} / \mathrm{N}$ in the FUV and NUV of the CASBaH spectra.

This paper is outlined as follows. Section 2 describes the galaxy selection criteria and the related photometry. Section 3

\footnotetext{
${ }^{12}$ For information on COS and STIS, see Green et al. (2012) and Woodgate et al. (1998) respectively.
} 
Table 1

Survey Overview

\begin{tabular}{|c|c|c|c|c|c|}
\hline Field & R.A. & Decl. & $z_{\mathrm{em}}$ & Imaging & Spectra \\
\hline PHL1377 & 38.78077 & -4.03491 & 1.437 & $\begin{array}{l}\text { SDSS/ugriz } \\
\text { LBT+LBC }\end{array}$ & $\begin{array}{c}\text { BOSS-DR12 } \\
\text { Keck/DEIMOS }\end{array}$ \\
\hline FBQS0751+2919 & 117.80128 & 29.32730 & 0.916 & $\begin{array}{l}\text { SDSS/ugriz } \\
\mathrm{LBT}+\mathrm{LBC}\end{array}$ & $\begin{array}{c}\text { BOSS-DR12 } \\
\text { MMT/Hectospec } \\
\text { Keck/DEIMOS }\end{array}$ \\
\hline PG1148+549 & 177.83526 & 54.62586 & 0.976 & $\begin{array}{l}\text { SDSS/ugriz } \\
\mathrm{LBT}+\mathrm{LBC}\end{array}$ & $\begin{array}{c}\text { BOSS-DR12 } \\
\text { MMT/Hectospec }\end{array}$ \\
\hline PG1206+459 & 182.24172 & 45.67652 & 1.165 & $\begin{array}{l}\text { SDSS/ugriz } \\
\mathrm{LBT}+\mathrm{LBC}\end{array}$ & $\begin{array}{c}\text { BOSS-DR12 } \\
\text { MMT/Hectospec } \\
\text { Keck/DEIMOS }\end{array}$ \\
\hline PG1338+416 & 205.25326 & 41.38724 & 1.214 & $\begin{array}{l}\text { SDSS/ugriz } \\
\text { LBT+LBC }\end{array}$ & BOSS-DR12 \\
\hline PG1407+265 & 212.34963 & 26.30587 & $0.94^{\mathrm{a}}$ & $\begin{array}{l}\text { SDSS/ugriz } \\
\mathrm{LBT}+\mathrm{LBC}\end{array}$ & $\begin{array}{c}\text { BOSS-DR12 } \\
\text { MMT/Hectospec } \\
\text { Keck/DEIMOS }\end{array}$ \\
\hline LBQS1435-0134 & 219.45118 & -1.78633 & 1.311 & SDSS/ugriz & BOSS-DR12 \\
\hline PG1522+101 & 231.10231 & 9.97494 & 1.328 & SDSS/ugriz & $\begin{array}{c}\text { BOSS-DR12 } \\
\text { MMT/Hectospec }\end{array}$ \\
\hline PG1630+377 & 248.00462 & 37.63055 & 1.479 & $\begin{array}{l}\text { SDSS/ugriz } \\
\mathrm{LBT}+\mathrm{LBC}\end{array}$ & $\begin{array}{c}\text { BOSS-DR12 } \\
\text { MMT/Hectospec } \\
\text { Keck/DEIMOS }\end{array}$ \\
\hline
\end{tabular}

Note.

${ }^{\text {a }}$ This QSO lacks typical emission lines (e.g., the Ly $\alpha$ emission line is almost undetectable), and the optical emission lines that are detected span a redshift range of $\approx 10,000 \mathrm{~km} \mathrm{~s}^{-1}$ (McDowell et al. 1995). Consequently, the redshift of this QSO is more uncertain than the redshifts of the other targets; McDowell et al. report a redshift uncertainty of \pm 0.02 .

presents the spectroscopy and redshift measurements and Section 4 lists estimates for several derived quantities (e.g., stellar mass). Lastly, Section 6 presents a clustering analysis of these galaxies with themselves and against the population of $\mathrm{O}$ VI absorbers along the CASBaH sightlines. Throughout the analysis we adopt the Planck15 cosmology, as encoded in the ASTROPY $^{13}$ package.

\section{Sample Selection}

\subsection{Overview}

The basis of our CASBaH galaxy survey is the fields surrounding the nine quasars observed for the project with $H S T$ (T. Tripp et al. 2019, in preparation). These quasars are presented in Table 1, where we also list the ancillary data available in the public domain (as of 2018 May 16) and those collected by our team. ${ }^{14}$ The quasar coordinates were taken from the Simbad database, and we adopt the QSO emission redshift measured from SDSS spectra by Hewett \& Wild (2010).

Given that the scientific goals of the CASBaH project include the analysis of gas from $z \sim 0$ to $z \sim 1$ (T. Tripp et al. 2019 , in preparation), we pursued galaxies to faint magnitude limits, i.e., much fainter than typical of public spectroscopic data sets (although any such data are included). In general, our approach was two-pronged: (i) we obtained spectra to the SDSS imaging limit over a wide field of view (FOV) using the Hectospec spectrometer (Fabricant et al. 2005) on the MMT

\footnotetext{
13 http://www.astropy.org/

${ }^{14}$ Numerous other public imaging survey data sets (e.g., WISE) also cover these fields and are not listed but are employed in our galaxy characterization. These did not inform the sample selection.
}

telescope, and (ii) we obtained deep Large Binocular Camera (LBC) imaging with the Large Binocular Telescope (LBT) and faint object spectra with the DEIMOS spectrometer (Faber et al. 2003) on the Keck II telescope over a narrower FOV. Each of these activities had basic requirements, e.g., SDSS imaging for MMT/Hectospec, visibility from Keck, etc. In addition, the data collected flowed from the vagaries of time assignment committees and weather. These factors resulted in more heterogeneous sampling of each field than may be desired.

\subsection{Photometry}

\subsubsection{SDSS}

For the eight fields within the SDSS imaging footprint, we retrieved the photometric measurements from their archive with the ASTROQUERY ${ }^{15}$ package. Specifically, we retrieved all photometric sources in the SDSS-DR12 catalog within $2^{\circ}$ of each field, requesting Petrosian magnitudes and errors. We then cross-matched these to the spectroscopic catalog and cut on $z>0.001666\left(v>500 \mathrm{~km} \mathrm{~s}^{-1}\right)$ to trim stars. ${ }^{16}$ These data form the primary public data set integrated within our database.

For the fields observed with Hectospec, we further queried the SDSS photometric catalogs to generate a set of targets. Again, we use Petrosian magnitudes and errors. A full description of the Hectospec targeting is given in Section 2.3.1.

\footnotetext{
15 http://github.com/astropy/astroquery

16 Galaxies at $v<500 \mathrm{~km} \mathrm{~s}^{-1}$ are difficult to use at any rate because the $\mathrm{H} \mathrm{I}$ Ly $\alpha$ line is lost within the Milky Way damped Ly $\alpha$ profile and the geocoronal Ly $\alpha$ emission, which affects a substantial region in COS spectra.
} 
Table 2

LBT/LBC Observing

\begin{tabular}{|c|c|c|c|}
\hline Field & Date & Seeing & Filters \\
\hline PKS0232-042 & 2010 Oct, 2010 Dec & 1.0 & $U B V I$ \\
\hline FBQS0751+2919 & 2010 Oct, 2010 Dec & 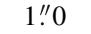 & $U B V I$ \\
\hline PG1148+549 & 2011 May & $0 ! .8$ & $U B V I$ \\
\hline PG1206+459 & 2010 May, 2010 Dec, 2011 May & $1 ! 0$ & $U B V I$ \\
\hline PG1338+416 & $2011 \mathrm{Apr}$ & $1 \stackrel{\prime \prime}{.}$ & $U B V I$ \\
\hline PG1407+265 & 2010 Mar & $1 !$ & $U B V I$ \\
\hline PG1522+101 & 2011 May & $0 ! " 8$ & $U B V I$ \\
\hline PG1630+377 & 2009 Jun & $1 \stackrel{\prime \prime}{0}$ & griz \\
\hline
\end{tabular}

\subsection{2. $L B T / L B C$}

For seven fields, we obtained deep multi-band (UBVI or griz) images with the LBC on LBT under a variety of conditions (PIs: Howk, Ford). Table 2 summarizes the observations. The two LBC cameras described by Giallongo et al. (2008) sit at the prime foci of the twin $8.4 \mathrm{~m}$ mirrors of the LBT. The blue $(U$, $B$ ) and red $(V, I)$ LBCs each provide a $23^{\prime}$ field of view using a four-chip mosaic. We used dithered observations (typically a nine-step dither pattern for these data) to fill in the inter-chip spacings, and we used twilight sky flats to perform flat-field corrections. Total exposure times are typically $3000 \mathrm{~s}$ for the $U$, $I$ band images and $420 \mathrm{~s}$ for the $B, V$ bands. For a subset of the images, one of the CCDs in the mosaic was unavailable (CCD \#3). In those cases we filled in the missing area with additional dithers, which provided additional exposure time for other areas of the field, so these exposure times should be taken as representative only.

The LBC data were reduced with a development version of the Python-based LBCgo data reduction pipeline (J. C. Howk et al. 2019, in preparation). ${ }^{17} \mathrm{LBCg}$ o performs basic image processing steps, such as removing the overscan strip, deriving and applying flat fields, etc., following standard practice. After basic image processing, LBCgo uses several Astromatic. net ${ }^{18}$ codes to project the images onto a common WCS frame and coadd them, following an approach described first by Sand et al. (2009). On a chip-by-chip basis (by default) LBCgo uses Source Extractor (Bertin \& Arnouts 1996) to find sources detected in each chip. It then uses SCAMP (Bertin et al. 2002) to derive the astrometric solution for each chip based after matching detected sources with the Gaia-DR1 catalog (Gaia Collaboration et al. 2016a, 2016b). The astrometric solution is critical given the distortions over the full $23^{\prime}$ LBC field. The individual chips are then resampled, background subtracted, and coadded using SWARP (Bertin et al. 2002). The astrometric solution provided by SCAMP has a typical reported rms $\sim 0$ " 05 , with values typically ranging from $\sim 0$ ". $03-0$ "! 10 per exposure.

We then adopted published zero-points for the instrument ${ }^{19}$ and corrected for airmass but not Galactic extinction: $U$ $($ SDT_USpec $)=27.33, \quad B \quad($ Bessel $)=27.93, \quad V \quad($ Bessel $)=27.94, \quad I \quad($ Bessel $)=27.59, \quad g \quad($ Sloan $)=28.31, \quad r$ $($ Sloan $)=27.75$. With our typical total exposure times, we achieved the sensitivities listed in Table 3. Figure 1 shows the $V$-band image of the field surrounding PG1407+265, which is typical of our full data set. Additional examples of the LBT

\footnotetext{
17 https://github.com/jchowk/LBCgo

18 http://www.astromatic.net/

19 http://abell.as.arizona.edu/ lbtsci/Instruments/LBC/lbc_description. html\#zero-points.html
}

Table 3

LBT/LBC Imaging

\begin{tabular}{lccc}
\hline \hline Field & Filter & AM $^{\mathrm{a}}$ & Mag. Limit $^{\mathrm{b}}$ \\
\hline PG1206+459 & $U$ & 1.3 & 27.42 \\
PG1206+459 & $B$ & 1.0 & 26.51 \\
PG1206+459 & $V$ & 0.9 & 26.20 \\
PG1206+459 & $I$ & 0.9 & 25.29 \\
PHL1377 & $U$ & 1.0 & 27.46 \\
PHL1377 & $B$ & 1.0 & 27.12 \\
PHL1377 & $V$ & 0.9 & 26.79 \\
PHL1377 & $I$ & 1.0 & 26.67 \\
PG1407+265 & $U$ & 1.0 & 27.62 \\
PG1407+265 & $B$ & 1.3 & 27.49 \\
PG1407+265 & $V$ & 1.4 & 27.10 \\
PG1407+265 & $I$ & 1.3 & 26.77 \\
PG1448+549 & $B$ & 1.0 & 26.50 \\
PG1448+549 & $U$ & 1.1 & 27.53 \\
PG1630+377 & $g$ & 1.1 & 28.08 \\
PG1630+377 & $r$ & 1.0 & 27.20 \\
\hline
\end{tabular}

Notes.

${ }^{\mathrm{a}}$ Median airmass of the observations.

${ }^{\mathrm{b}}$ The $5 \sigma$ limiting magnitude for a point source is computed by finding the mean value across all images in a given filter of the faintest sources whose magnitude error is $0.198 \mathrm{mag}$ or less using the SExtractor MAG_AUTO parameter. SExtractor is run with a detection threshold of a $2.5 \sigma$ point source with at least 3 pixels above the threshold.

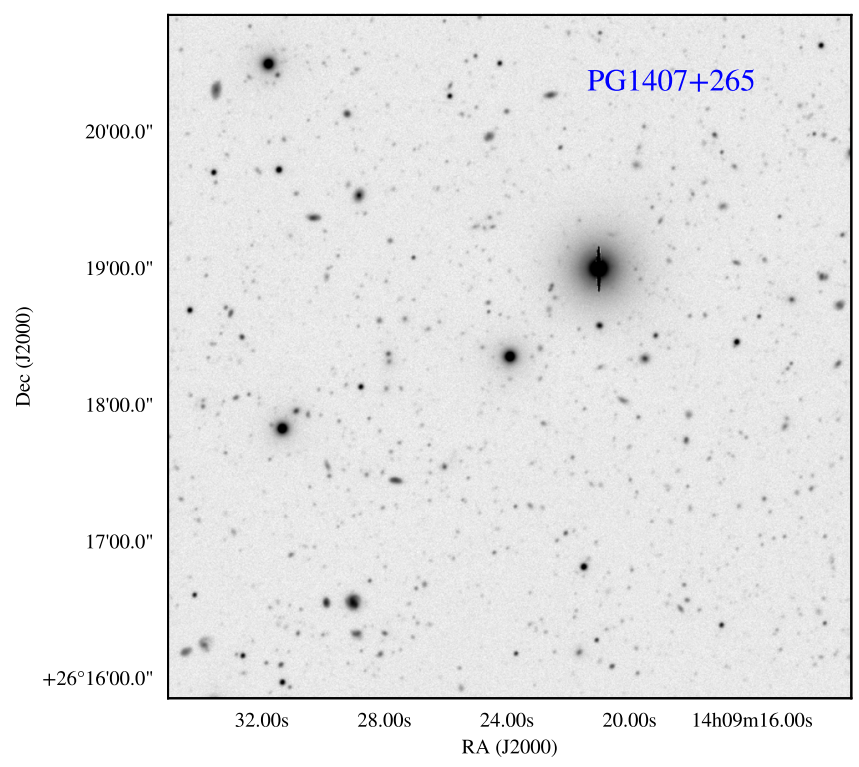

Figure 1. $V$-band image of the field surrounding PG1407+265 (centered) obtained with the LBC on the LBT.

imaging are presented in Ribaudo et al. (2011b), Tripp et al. (2011), Meiring et al. (2013), and Burchett et al. (2013); these examples are more zoomed-in and thus show the depth and morphological information provided by the LBT imaging in greater detail.

On each of the reduced images, we ran the SExtractor software package to generate a catalog of sources. We adopted a standard parameter suite, including the following extra parameters: CLASS_STAR, A_IMAGE, B_IMAGE, THETA_IMAGE and MU_THRESHOLD. The image parameters are included in the database, although we caution that the uncertainties are large for faint and/or compact sources. For 


\section{PG1407+265: MMT/Hectospec Targets}
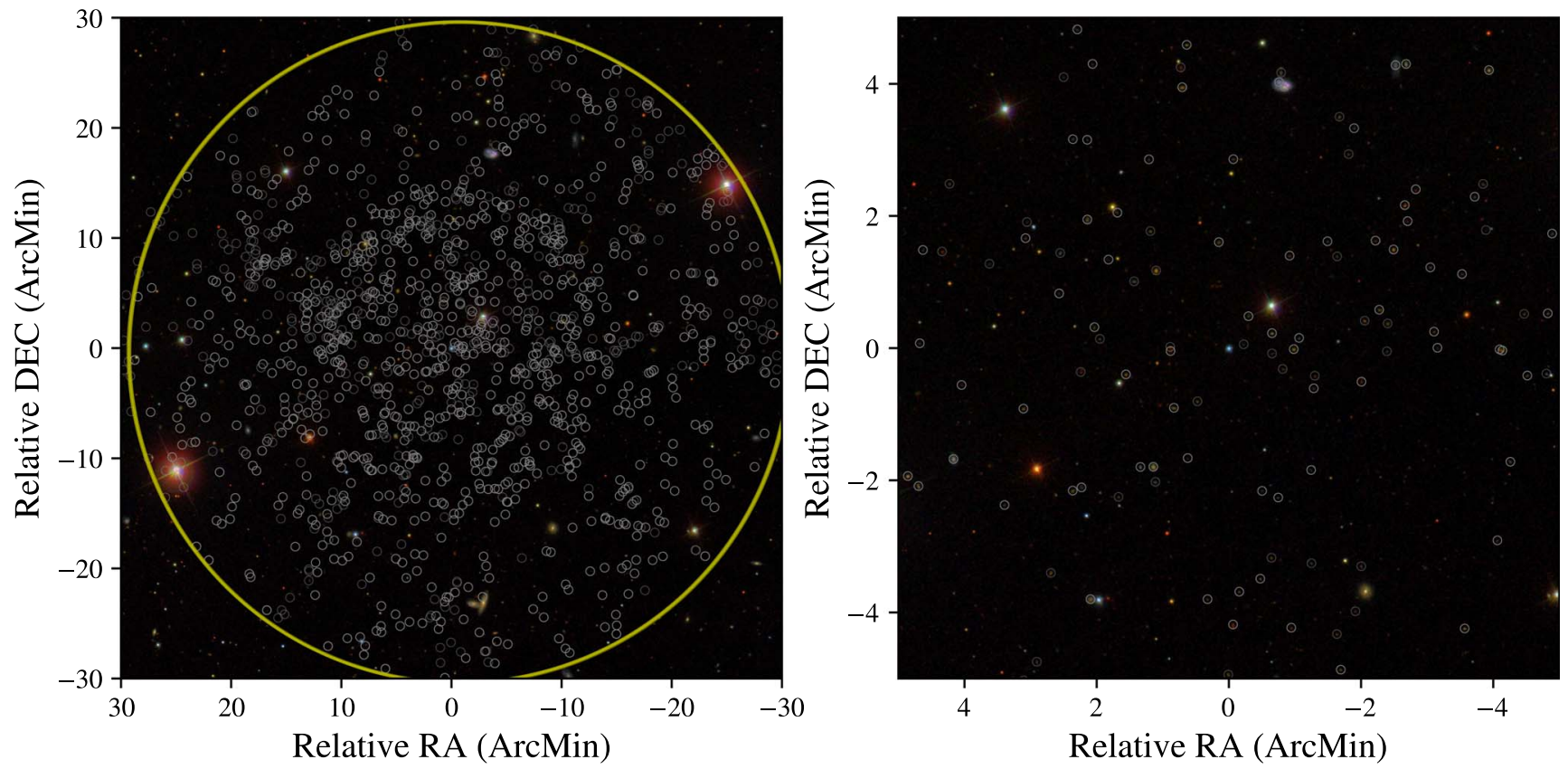

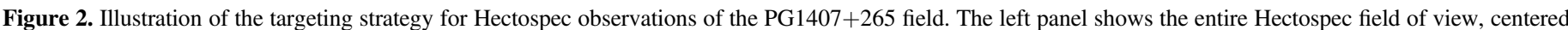

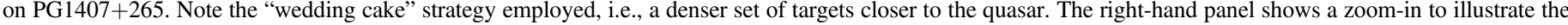
depth of targeting.

source detection, we adopted three pixels as the minimum number of pixels above a detection threshold of $2.5 \sigma$. Each filtered image was processed separately and sources were crossmatched in custom software based on the astrometry.

\subsection{Targeting \\ 2.3.1. Hectospec}

From the SDSS imaging data described above, we generated target lists to observe with the MMT/Hectospec spectrograph employing a "wedding-cake" strategy that sampled the inner angular offsets from the quasar to fainter magnitudes. Specifically, we targeted galaxies with $r<22$ mag for $\theta<5^{\prime}, r<21 \mathrm{mag}$ for $\theta=\left[5^{\prime}, 10^{\prime}\right]$, and $r<20 \mathrm{mag}$ to $\theta=30^{\prime}$. This was done because at lower redshifts, it is desirable to cover a larger FOV to probe similar impact parameter ranges as the higher- $z$ data, but the survey does not need to go as deep as the higher- $z$ observations to reach similar galaxy luminosities.

Figure 2 shows an example of target selection taken from the PG1407+265 field and Figure 3 presents the completeness for all of the fields with the wedding-cake criterion above. The targeting completeness reported here is the percentage of targets with a fiber placed upon them during our observing runs. Details on the spectroscopy and redshift determinations are provided in the following section.

\subsubsection{DEIMOS}

With Keck/DEIMOS, we pursued fainter galaxies that are more effectively surveyed with this telescope/instrument combination, which has a smaller FOV but also a larger primary mirror. For these observations, we again gave higher priority to sources close to the quasar (in angular offset) but had additional, observing-related criteria that further affected our

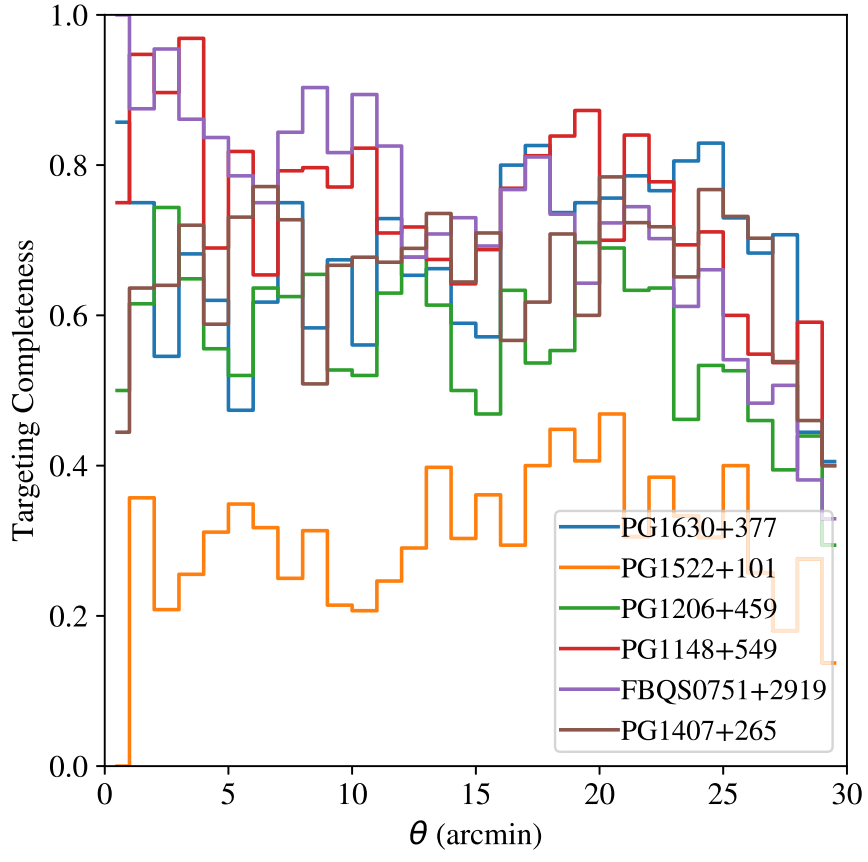

Figure 3. Completeness fraction for the targeting of galaxies (i.e., fraction of fibers on desired galaxies for Hectospec only) selected from SDSS imaging according to our targeting criteria. The value is independent of whether a precise redshift was measured from the resultant spectrum. Note that the sparsest field (PG1522+101) was observed with only two configurations.

slit-mask designs. Figure 4 illustrates the targeting for the PG1407 +265 field, where we adopted the following criteria: (1) $\theta<15^{\prime}$, (2) $14<V<24.5$, and (3) SExtractor star-galaxy classifier $\mathrm{S} / \mathrm{G}<0.9$, except for sources within $20^{\prime \prime}$ of the quasar where no $\mathrm{S} / \mathrm{G}$ criterion was applied. Other fields had small differences from these criteria, as summarized in Table 4. 


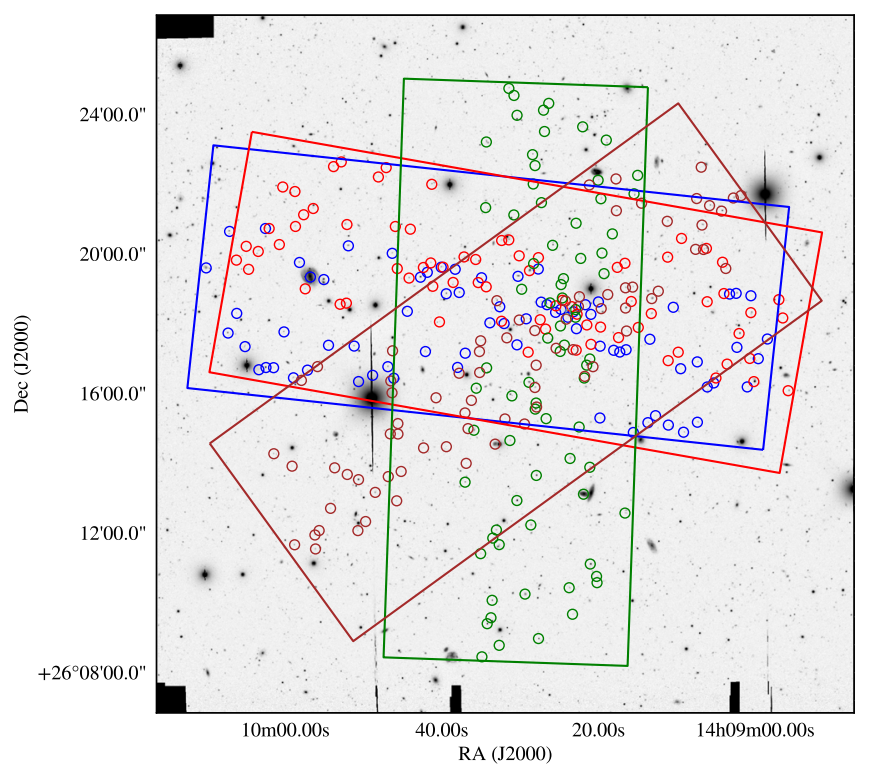

Figure 4. Slitmask fields of view (rectangles) and associated targets (circles) for the PG1407+265 field observed with Keck/DEIMOS. This quasar is surrounded by the densest distribution of targets among those in our survey.

Table 4

DEIMOS Targeting Criteria

\begin{tabular}{lcccc}
\hline \hline Field & Filter & $\mathrm{S} / \mathrm{G}_{\max }{ }^{\mathrm{a}}$ & $m_{\max }{ }^{\mathrm{b}}$ & $\theta_{\max }{ }^{\mathrm{c}}$ \\
\hline PG1630+377 & $R$ & 0.9 & 25.0 & 15.0 \\
PG1206+459 & $V$ & 0.9 & 25.0 & 15.0 \\
FBQS0751+2919 & $V$ & 1.0 & 24.5 & 10.0 \\
PG1407+265 & $V$ & 0.9 & 24.5 & 15.0 \\
PHL1377 & $V$ & 1.0 & 24.5 & 15.0 \\
\hline
\end{tabular}

Notes.

${ }^{a}$ Star/galaxy classifier from SExtractor with $\mathrm{S} / \mathrm{G}=1$ a star-like object. Ignored for $\theta<20$.

${ }^{\mathrm{b}}$ Faintest magnitude source for targeting.

${ }^{c}$ Largest angular offset for targeting.

In general, we avoided targeting sources previously observed by SDSS or our own Hectospec program. Similar to Figure Figure 3, 5 shows the completeness for the DEIMOS fields. In contrast to the Hectospec survey, the DEIMOS survey has sparser coverage and higher incompleteness, which resulted mainly from poor weather.

\section{Spectroscopy \\ 3.1. Observations \\ 3.1.1. Hectospec}

For all of the Hectospec data collected in our CASBaH survey, we employed an identical setup of 3001 1".5 fibers and the G270 grating, yielding $R \approx 1000$ with wavelength coverage $\lambda_{\mathrm{ob}} \approx 3700-9200 \AA$. The observations used three or more exposures with times ranging from 900 to $1800 \mathrm{~s}$ each, for a total exposure of $3600 \mathrm{~s}$ or $5400 \mathrm{~s}$ as detailed in Table 5. Each fiber configuration included $\sim 20$ fibers placed on "blank" sky.

All of these spectra were reduced by the HSREDv2 ${ }^{20}$ data reduction pipeline to wavelength calibrate, extract, sky

\footnotetext{
${ }^{20}$ http://www.mmto.org/node/536
}

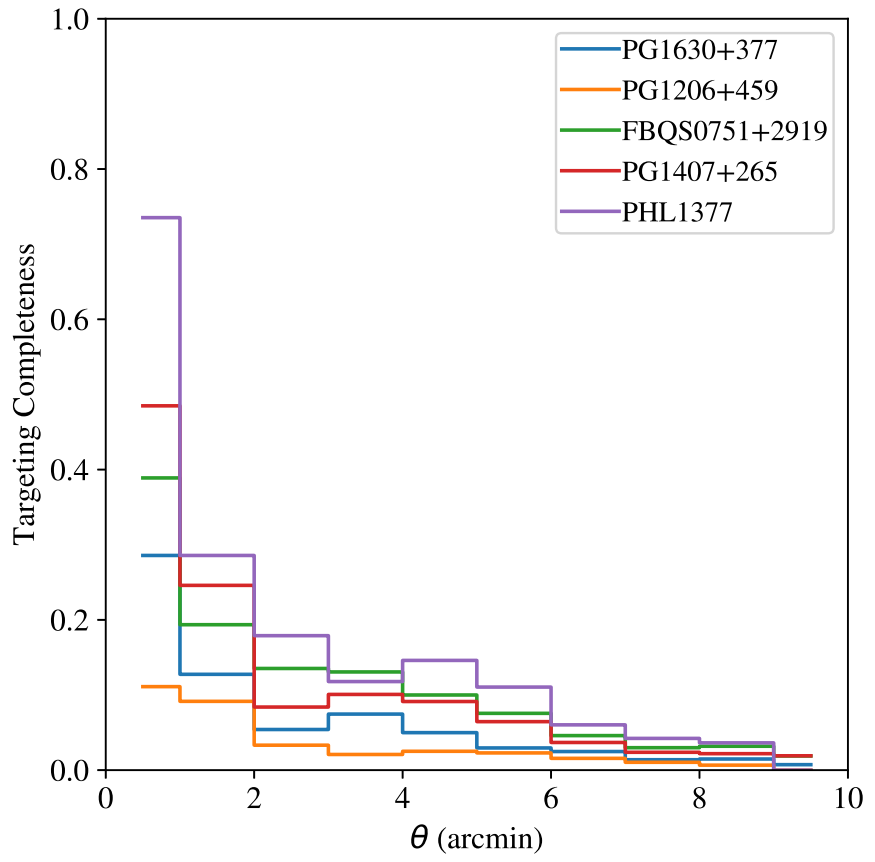

Figure 5. Completeness of targeting for the fields observed with Keck/ DEIMOS defined as the fraction of targets observed. The value is independent of whether a precise redshift was measured from the resultant spectrum.

Table 5

Hectospec Observing

\begin{tabular}{lccc}
\hline \hline Field & Configuration & Date & $\begin{array}{c}\text { Total Exposure } \\
(\mathrm{s})\end{array}$ \\
\hline FBQS0751+2919 & 1 & 2011 Jan 25 & 5400 \\
FBQS0751+2919 & 2 & 2011 Jan 26 & 5400 \\
FBQS0751+2919 & 3 & 2011 Feb 10 & 5400 \\
FBQS0751+2919 & 4 & 2011 Mar 25 & 5400 \\
& & & \\
PG1148+549 & 1 & 2011 Feb 10 & 5400 \\
PG1148+549 & 2 & 2011 Feb 11 & 5400 \\
PG1148+549 & 3 & 2011 Feb 11 & 5400 \\
PG1148+549 & 4 & 2011 Mar 30 & 7187 \\
& & & \\
PG1206+459 & 1 & 2012 Jan 21 & 3600 \\
PG1206+459 & 2 & 2012 Mar 14 & 3600 \\
PG1206+459 & 3 & 2012 Feb 22 & 3600 \\
& & & \\
PG1407+265 & 1 & 2011 Jun 3 & 3600 \\
PG1407+265 & 2 & 2011 Jun 3 & 3600 \\
PG1407+265 & 3 & 2011 Jun 5 & 3600 \\
PG1407+265 & 4 & 2011 Jun 6 & 3600 \\
& & & \\
$P G 1522+101$ & 1 & 2011 Jun 5 & 3600 \\
$P G 1522+101$ & 2 & 2012 Feb 22 & 3600 \\
& & & \\
$P G 1630+377$ & 1 & 2011 Jun 3 & 3600 \\
$P G 1630+377$ & 2 & 2011 Jun 4 & 2700 \\
$P G 1630+377$ & 3 & 2011 Jun 5 & 3120 \\
$P G 1630+377$ & 4 & 2011 Jun 6 & 3600 \\
\hline & & &
\end{tabular}

subtract, and flux the fiber data. The $1 \sigma$ error array assumes Gaussian statistics and a two-electron read noise term. Each exposure was reduced separately, and the final 1D spectra were coadded in wavelength space weighted by the inverse variance of the individual exposures. The pipeline, in our case, produced a wavelength solution calibrated in air and unfluxed. Therefore, 

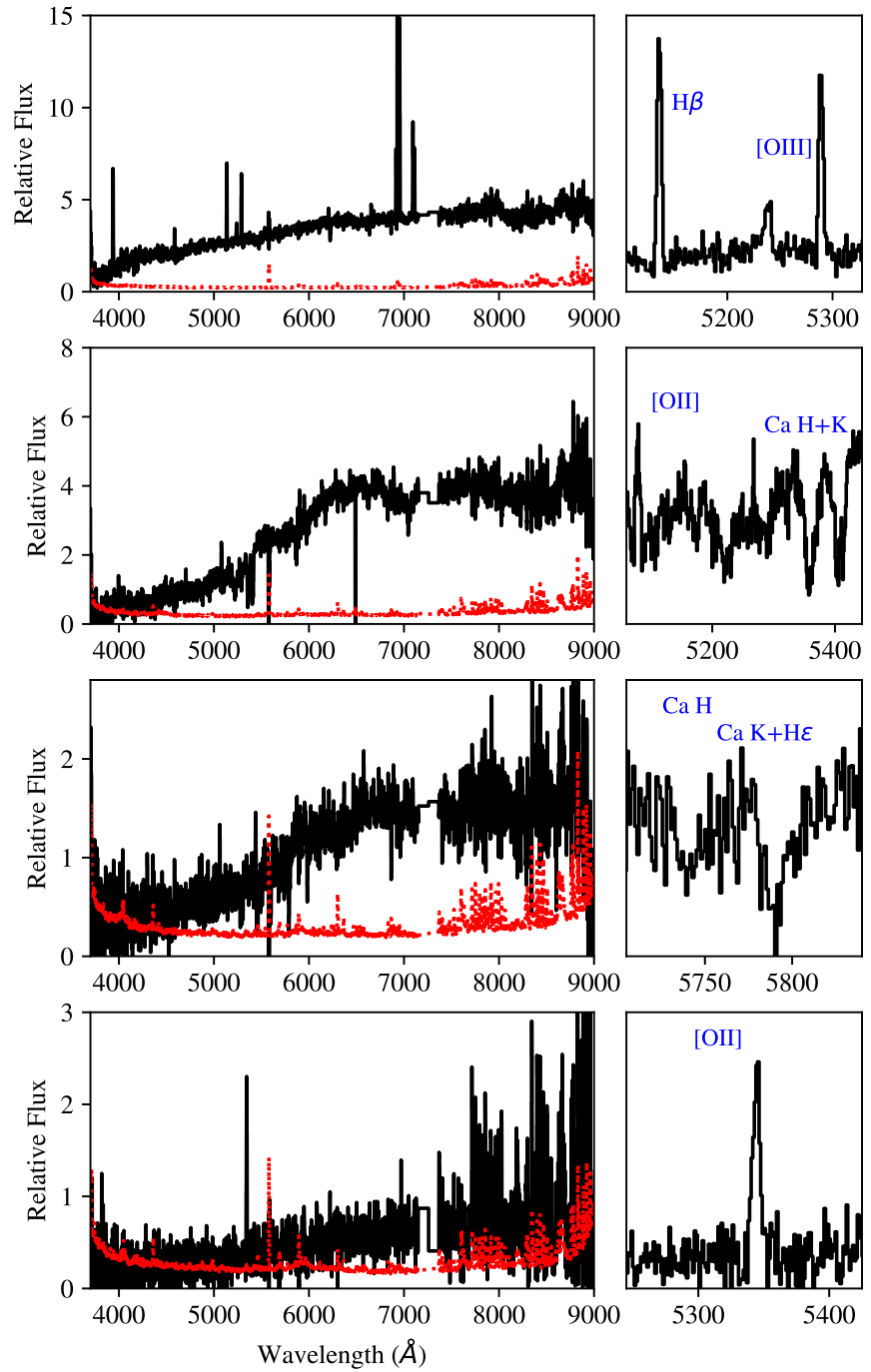

Figure 6. Representative spectra from the Hectospec observations. Cut-outs on the right show examples of key spectral features used in the redshift determination.

we converted to vacuum with the Ciddor equation described at NIST. ${ }^{21}$ The spectra were fluxed using a sensitivity function derived from Feige 34 observed on another program. We caution that the absolute fluxes do not include corrections for fiber losses, airmass, telluric absorption, or variable observing conditions.

Figure 6 shows several examples of spectra for sources spanning the dynamic range of observed magnitudes $(r \approx 18.1-21.9 \mathrm{mag})$. The median $\mathrm{S} / \mathrm{N}$ of all spectra is approximately 5.3 per $1.2 \AA$ pixel at $\lambda_{\text {ob }} \approx 5000 \AA$.

\subsubsection{DEIMOS}

For the DEIMOS observations of the CASBaH target fields (Table 6), we designed slitmasks with the DSIMULATOR software taking into account atmospheric dispersion with an attempt to optimize targets and observing time. We employed the G600 grating, which yields a spectral resolution $R \approx 1600$ for our $1^{\prime \prime}$ slits, a dispersion of $\approx 0.5 \AA$ per pixel, and an approximate wavelength coverage of $\lambda_{\mathrm{ob}} \approx 5000-10,000 \AA$. The spectral images include arc and quartz lamp calibration

$\overline{21}$ https://emtoolbox.nist.gov/Wavelength/Documentation.asp
Table 6

DEIMOS Spectroscopic Observations

\begin{tabular}{lccc}
\hline \hline Field & Mask ID & $N_{\text {slitlets }}$ & Date Obs $^{\text {a }}$ \\
\hline PHL1377 & M1-8005 & 88 & 2012 Nov 14 \\
PHL1377 & M2-8006 & 87 & 2012 Nov 14, Dec 13 \\
PHL1377 & M3-8007 & 86 & 2012 Nov 14 \\
FBQS0751+2919 & M4-8008 & 87 & 2012 Nov 14, Dec 13 \\
FBQS0751+2919 & M5-8009 & 93 & 2012 Dec 13 \\
PG1206+459 & M1-8377 & 72 & 2013 May 7, 8 \\
PG1407+265 & M1-8380 & 75 & 2013 May 7, 8 \\
PG1407+265 & M2-8381 & 74 & 2013 May 8 \\
PG1407+265 & M3-8382 & 74 & 2013 May 8 7,8 \\
PG1407+265 & M4-8383 & 76 & 2013 May 7 \\
PG1630+377 & M3-8387 & 69 & 2013 May 8
\end{tabular}

Note.

${ }^{a}$ Exposure times achieved on each mask were $4 \times 1800 \mathrm{~s}$ exposures, sometimes spanning two nights.

frames. All of these data were reduced with the SPEC2D data reduction pipeline developed by $\mathrm{M}$. Cooper for the DEEP survey (Newman et al. 2013). The pipeline produces optimally extracted, wavelength-calibrated spectra (in air and converted later to vacuum). Multiple exposures taken with a given mask on the same night are combined in $2 \mathrm{D}$ by the data reduction pipeline (DRP). Masks exposed on separate nights were extracted separately and the individually, extracted 1D-spectra were coadded with a custom algorithm.

From observations of two spectrophotometric standards, Feige 110 and 34, on three separate nights, 2012 November 14, 2012 December 13 (Feige 110), and 2013 May 8 (Feige 34) we generated a combined sensitivity function that was applied to the entire CASBaH DEIMOS spectroscopic data set. Again, we made no correction for slit losses, airmass, or variable observing conditions. Furthermore, vignetting at the edges of the detector leads to fluxing error at the longest and shortest wavelengths.

Representative spectra spanning the dynamic range of sources observed with DEIMOS are illustrated in Figure 7. The median $\mathrm{S} / \mathrm{N}$ of the complete data set at $\lambda_{\mathrm{ob}} \approx 6500 \AA$ is $\approx 2.9$ per 0.6 A pixel.

The standard mode of the SPEC2D extraction algorithm searches for additional sources in each slit (in part to assist sky subtraction) and extracts them. We have used the mask information and our astrometry to assign each an R.A. and decl. coordinate. Where possible, we have also matched them to our photometric catalog. All of these "serendipitous" spectra are included in the database and were analyzed in a similar manner to the primary targets.

\subsection{Redshift Analysis}

Redshift analysis proceeded in two stages. The first stage employed a template-fitting algorithm custom to the spectrograph (see the following sections). These results were vetted by one or more co-authors and a quality flag $Z_{Q}$ was assigned to each source with $Z_{Q}=0,1,2,3,4$ as follows: (0) data too poor for any assessment; (1) data poor and redshift highly uncertain; (2) data quality good but no redshift determined; (3) data quality good and redshift is highly likely but not confirmed by multiple lines (or one line is of low $S / N$ ); 

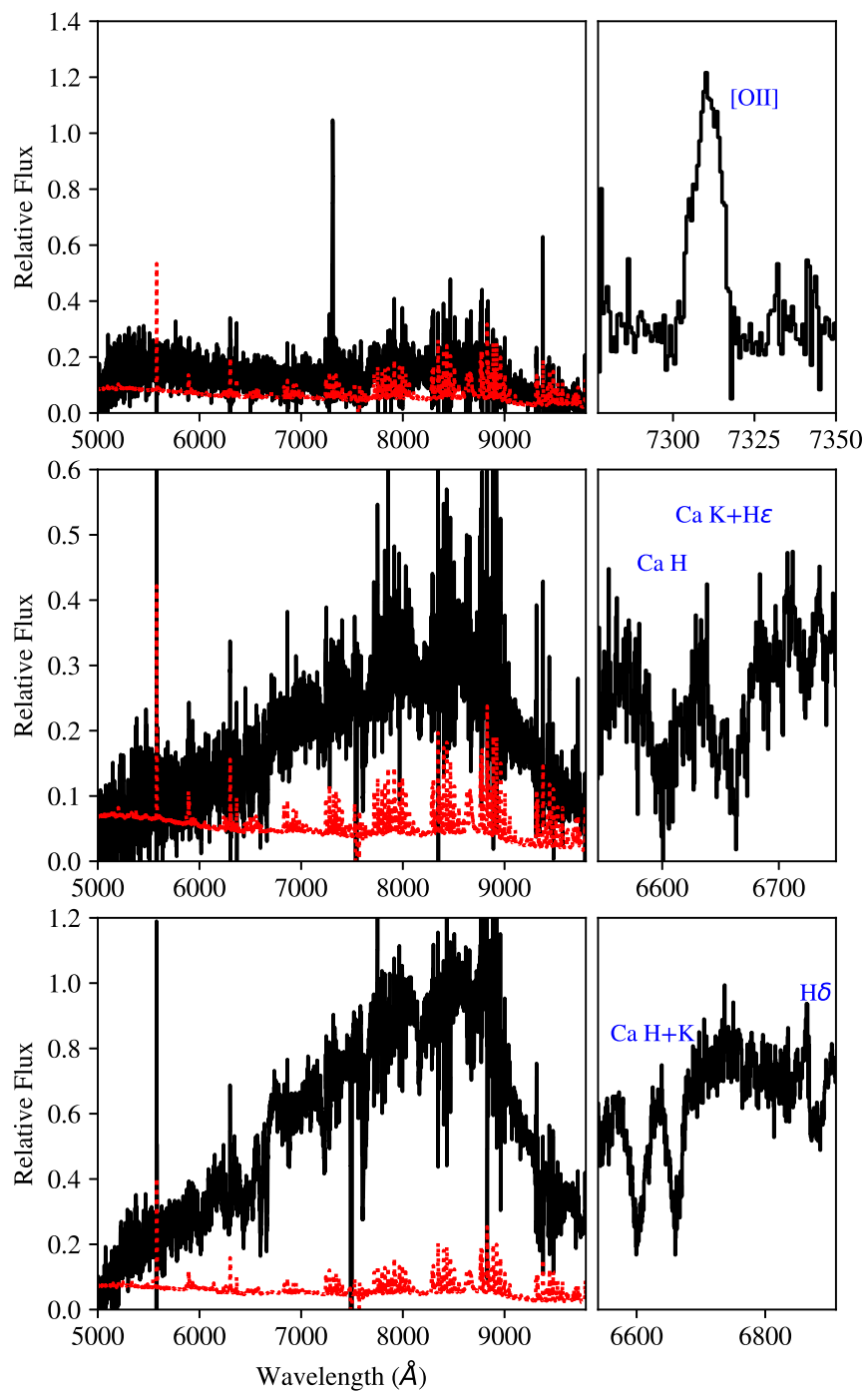

Figure 7. Gallery of example spectra from the Keck/DEIMOS observations, ordered from top to bottom by increasing flux. Cut-outs on the right show examples of key spectral features used in the redshift determination.

(4) highly certain and confirmed by multiple spectral features (see also Newman et al. 2013). We then analyzed each spectrum with the REDROCK software package ${ }^{22} \mathrm{v} 0.7$, which is under development by the Dark Energy Spectroscopic Instrument (DESI) project. This code also compares a set of galaxy and star templates to the spectra to generate a set of best-fitting models and corresponding redshift estimates. We then inspected every REDROCK solution offset by more than $50 \mathrm{~km} \mathrm{~s}^{-1}$ from the original estimate and resolved the conflict based on the observed spectral features (if any). Only sources with $Z_{Q} \geqslant 3$ are considered reliable. We now detail specific aspects of the analysis and results for each instrument.

\subsubsection{Hectospec}

The first algorithm adopted for Hectospec redshift calculation is a modified version of ZFIND developed by the SDSS project, which cross-correlates a series of templates (stars, galaxies, and quasars) in measurement space (i.e., no Fourier transform is applied). These are then ranked in $\chi^{2}$ and the

\footnotetext{
${ }^{22}$ https://github.com/desihub/redrock
}

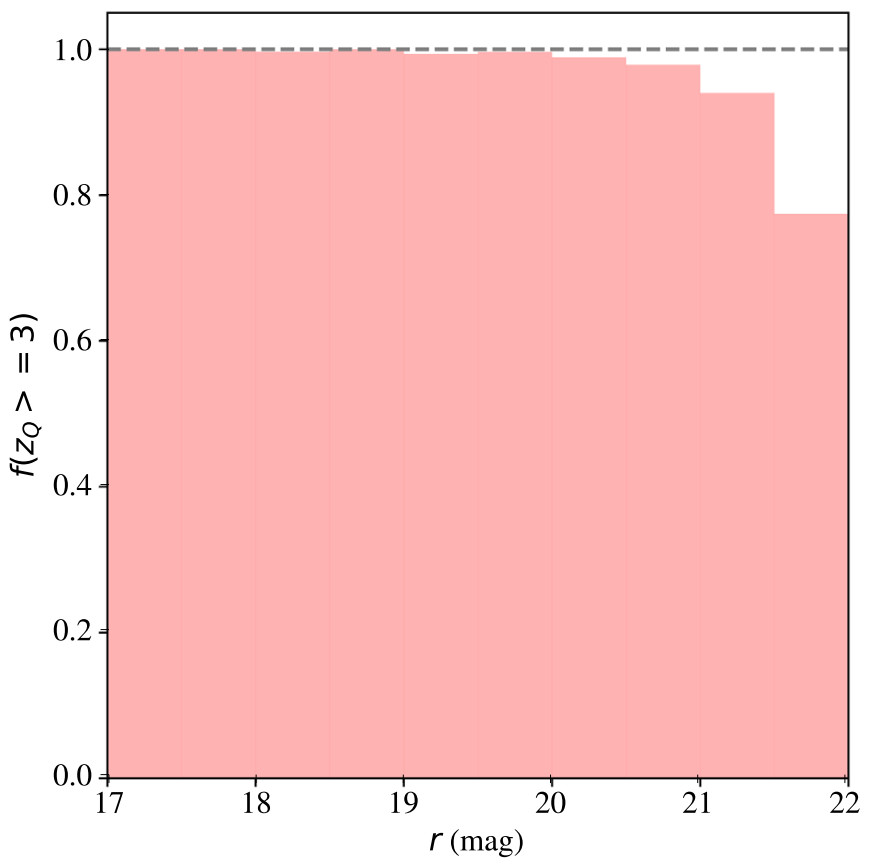

Figure 8. Redshift completeness fraction for sources observed with Hectospec as a function of $r$ magnitude.

lowest value is selected by the redshift code. Figure 8 summarizes the fraction of sources with $Z_{Q} \geqslant 3$ as a function of observed magnitude. For $r<21 \mathrm{mag}$, the redshift success rate exceeded $95 \%$ and, as expected, declined with fainter sources. Nevertheless, the success rate remains high to the magnitude-limit of the survey.

Spectra that showed redshift discrepancies of the order of $50 \mathrm{~km} \mathrm{~s}^{-1}$ between the REDROCK and Hectospec solutions were visually inspected, and the solution that showed the best matching locations of certain prominent galaxy lines was selected. We found that the majority of cases favored the REDROCK determination, though in cases where the $\mathrm{S} / \mathrm{N}$ was low, the Hectospec/ZFIND determination frequently showed the higher confidence redshift.

The reported redshift uncertainty from these $\chi^{2}$-minimization codes is frequently several times $10^{-5}$, i.e., $\sigma_{v} \approx 10 \mathrm{~km} \mathrm{~s}^{-1}$. We consider such small errors to be overly optimistic and suggest one assume a minimum of $30 \mathrm{~km} \mathrm{~s}^{-1}$ uncertainty due to systematic error (e.g., wavelength calibration).

\subsubsection{DEIMOS}

For the DEIMOS spectra, we derived redshifts with two separate algorithms with two different sets of co-authors: (i) a modified version of the SDSS ZFIND algorithm by J.W. and S. L. and (ii) the REDROCK software package v0.7 developed by the DESI experiment. For nearly 500 sources, the two packages reported redshifts that matched to within $50 \mathrm{~km} \mathrm{~s}^{-1}$. For these we simply adopted the REDROCK estimate and assigned $Z_{Q}=3$. For all other cases (approximately 600 sources, the majority of which had no previously determined redshift), we vetted each of these manually. After visually inspecting the spectra, we assigned the solution with coincident, prominent spectral features. In a few percent of the cases, we assigned a redshift of our own estimation. This included most of the 


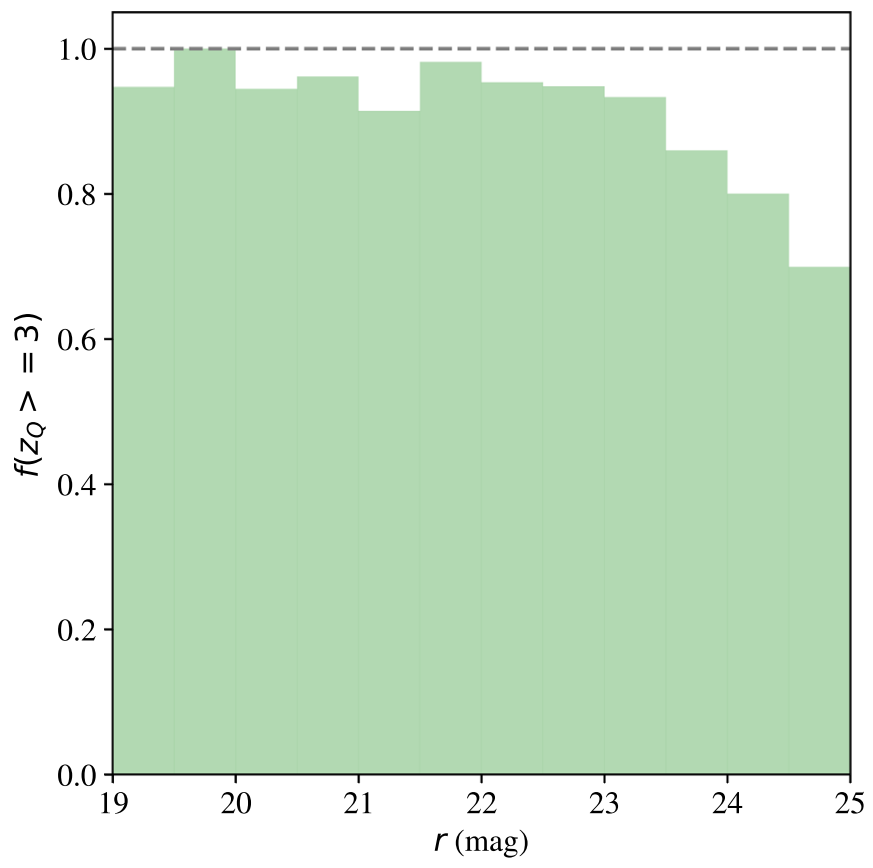

Figure 9. Redshift success rate for sources observed with DEIMOS as a function of $r$ magnitude.

galaxies at $z>1$ because we had limited our automatic search to less than this redshift.

Figure 9 shows the redshift measurement success rates from our DEIMOS analysis where a secure redshift is defined with $Z_{Q} \geqslant 3$. This analysis is limited to the primary targets, i.e., we ignore serendipitous sources that entered the DEIMOS slits. The success rate is approximately $95 \%$ to $22 \mathrm{mag}$, declines to $\approx 80 \%$ at $24 \mathrm{mag}$, and drops rapidly from there.

\subsection{Galaxy Spectroscopy Summary}

Integrating the redshift measurements from Hectospec, DEIMOS, and the SDSS database, we performed internal comparisons between the $\sim 175$ sources common to two or more of the sub-surveys. Ignoring catostrophic failures (described below), the measured rms values between Hectosec/SDSS and DEIMOS/Hectospec are $\approx 35 \mathrm{~km} \mathrm{~s}^{-1}$ and $\approx 36 \mathrm{~km} \mathrm{~s}^{-1}$ respectively. Therefore, we advise adopting a minimum redshift uncertainty of $35 \mathrm{~km} \mathrm{~s}^{-1}$ for galaxies drawn from the $\mathrm{CASBaH}$ database. We find no variation with emission/absorption-line properties among this common set of galaxies. This exercise also revealed 13 cases where the recovered redshifts were substantially offset $\left(\delta v \gg 100 \mathrm{~km} \mathrm{~s}^{-1}\right)$. In nearly all of the cases, at least one of the two spectra was of poor data quality. All but one of the nonSDSS spectra already showed $Z_{Q}<3$ and we have now downgraded the few from SDSS accordingly. In two cases, there were multiple sources with separation $<1^{\prime \prime}$ in the slit/ fiber; we have corrected the catalog accordingly.

The CASBaH spectroscopic redshift survey is summarized in Table 7. Altogether in the nine fields, we have 5902 galaxies with high-quality redshifts $\left(Z_{Q} \geqslant 3\right)$ and $z_{\mathrm{em}}>0.00166$. Their redshift distribution is shown in Figure 10 for the three primary data sets of our program. ${ }^{23}$ Clearly, our Hectospec survey

\footnotetext{
23 The full database includes several sources observed with Keck/LRIS, several with MMT/BCS, and a small set of galaxies from Johnson et al. (2015) that they kindly made public.
}

provides the majority of spectroscopic redshifts. These lie predominantly at $z_{\mathrm{em}}=[0,0.5]$. The DEIMOS data set contributes primarily at $z_{\mathrm{em}}>0.5$, as designed. We also report 356 sources with $Z_{Q}<3$ and no secure redshift measured as well as 279 spectra from DEIMOS and Hectospec of stars.

With our adopted cosmology, we may translate the galaxy redshifts and their angular offsets from their corresponding quasar sightline to estimate the impact parameter (physical $R_{\text {phys }}$ and comoving $\left.R_{\text {com }}\right)$. The distributions on small $\left(R_{\text {phys }}<450 \mathrm{kpc}\right)$ and large $\left(R_{\text {com }} \sim 10 \mathrm{cMpc}\right)$ scales are summarized in Figure 11. These distributions show the statistical power of CASBaH for studies of the CGM and IGM, respectively.

\section{Derived Quantities}

The previous sections described measurements made (nearly) directly from the imaging and spectroscopic data. This section describes a few quantities derived from the combined measurements, e.g., two or more filters and/or a combination of photometry and spectroscopy. We generally adopt standard techniques, assumptions, and software in the analysis and warn that the uncertainties (especially systematic error) can be large.

\subsection{Spectral Energy Distribution Fitting}

To estimate the stellar masses and star formation rates (SFRs) of all galaxies in our database, we have employed our spectroscopic redshifts, the photometric measurements from our own LBT/LBC observations, and photometry from various publicly available survey catalogs spanning the optical and near-infrared. We fit the spectral energy distributions (SEDs) of each galaxy with stellar population model spectra, models for dust attenuation, and emission from nebular lines using the CIGALE software package (Noll et al. 2009). While myriad high-quality SED fitting codes are available, we chose CIGALE for several reasons, including its ability to easily handle our heterogeneous data set given the variety of sources from which our data are derived (e.g., certain fields were covered by a given survey while others were not, and within a field not all objects were detected in a single survey). Furthermore, CIGALE natively supports several choices for stellar-population models, dust models, etc., across a wide range of parameters. We discuss our choices for these parameters below so that the reader may recreate or improve upon our estimates.

We assembled our photometric data set for SED fitting by cross-matching all galaxies in our spectroscopic database having redshift quality scores $Z_{Q} \geqslant 3$ with several public survey catalogs: SDSS DR12 (Alam et al. 2015, ugriz coverage of all fields except PHL1377), the Canada-France-Hawaii Telescope Legacy Survey (CFHTLS; Hudelot et al. 2012, ugriz coverage of the PHL1377 field), the WISE all sky survey (Cutri et al. 2013, 3.4, 4.6, 12, and $22 \mu \mathrm{m}$ bands for all fields), BASS (Zou et al. 2017a, 2017b), UKIDSS (Lawrence et al. 2007; Warren et al. 2007), KiDS (de Jong et al. 2017), DECALS (Dey et al. 2019), MzLS (Dey et al. 2019), and Pan-STARRS (Chambers et al. 2016; Flewelling et al. 2016).

We then corrected all magnitudes for Galactic reddening using the Schlafly \& Finkbeiner (2011) extinction values 
Table 7

CASBaH Redshift Survey

\begin{tabular}{|c|c|c|c|c|c|c|}
\hline CAS_ID & R.A. & Decl. & $z_{\mathrm{em}}$ & $\sigma(z)^{\mathrm{a}}$ & ZQ & Instr. \\
\hline 4494 & 212.37009 & 26.32546 & 0.3273 & 0.0001 & 4 & SDSS \\
\hline 4495 & 212.38848 & 26.47184 & 0.2964 & 0.0001 & 4 & SDSS \\
\hline 4496 & 212.31438 & 26.36133 & 0.0000 & 0.0001 & 3 & Hectospec \\
\hline 4497 & 212.45230 & 26.21947 & 0.5490 & 0.0001 & 3 & Hectospec \\
\hline 4498 & 212.39738 & 26.31966 & 0.8081 & 0.0001 & 3 & Hectospec \\
\hline 4499 & 212.38745 & 26.31115 & 0.3975 & 0.0001 & 3 & Hectospec \\
\hline 4500 & 212.46825 & 26.34760 & 0.4230 & 0.0001 & 4 & Hectospec \\
\hline 4501 & 212.25992 & 26.31462 & 0.3698 & 0.0001 & 3 & Hectospec \\
\hline 4502 & 212.28271 & 26.31439 & 0.0000 & 0.0001 & 3 & DEIMOS \\
\hline 4503 & 212.45042 & 26.33128 & 0.0000 & 0.0001 & 3 & DEIMOS \\
\hline 4504 & 212.46587 & 26.30656 & 0.0000 & 0.0001 & 3 & DEIMOS \\
\hline 4505 & 212.47700 & 26.31067 & 0.0000 & 0.0001 & 3 & DEIMOS \\
\hline 4506 & 212.35095 & 26.31103 & -1.0000 & 0.0001 & 0 & DEIMOS \\
\hline 4507 & 212.28904 & 26.34083 & 0.4264 & 0.0001 & 3 & DEIMOS \\
\hline 4508 & 231.20121 & 9.91829 & 0.6041 & 0.0002 & 3 & SDSS \\
\hline 4509 & 231.19939 & 9.93566 & 0.1518 & 0.0001 & 3 & SDSS \\
\hline
\end{tabular}

Note.

${ }^{\mathrm{a}}$ We limit the error to a minimum of $10^{-4}$ and advise readers to adopt a minimum uncertainty of $35 \mathrm{~km} \mathrm{~s}^{-1}$.

(This table is available in its entirety in machine-readable form.)

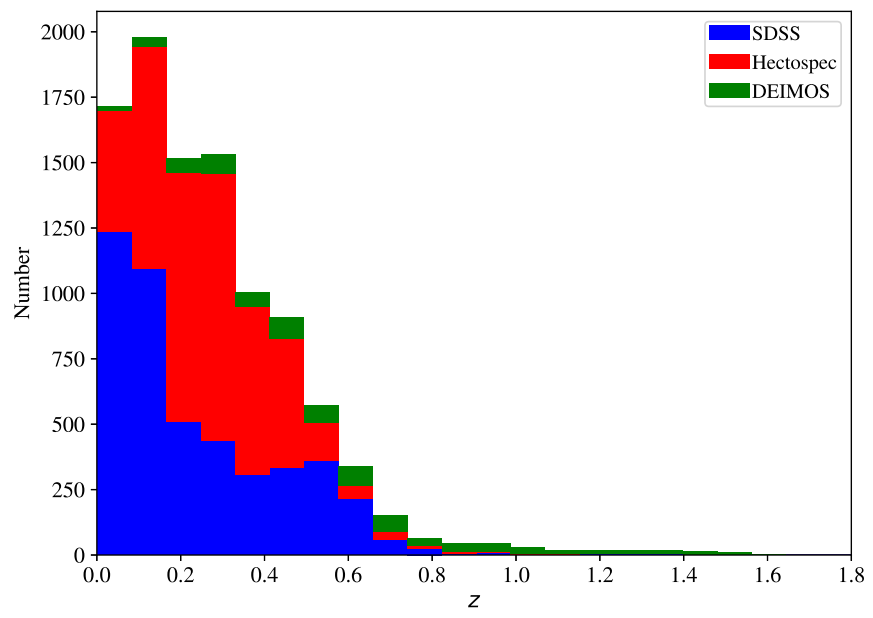

Figure 10. Histogram of the galaxy redshifts from the three main sources for the CASBaH survey: SDSS, Hectospec, and DEIMOS. The histograms are stacked, not overlapping, i.e., the DEIMOS histogram is placed above Hectospec.

provided by the Nasa Extragalactic Database ${ }^{24}$ service accessed through the ASTROQUERY framework. Reddening values are returned for a limited number of filter bandpasses (UBVRIugrizJHKL'), and we dereddened our photometry data using those most closely matching the central wavelengths of filters used in our compiled data set. CIGALE integrates fitted stellar population models over any filter response curve provided; we employed the SVO Filter Profile Service (Rodrigo et al. 2012) as well as individual survey websites to obtain filter curves and zero-points corresponding to each band.

As mentioned above, CIGALE includes a variety of models to include in the fitting. For stellar populations, we used the Bruzual \& Charlot (2003) models, assuming a Chabrier (2003) initial mass function, with metallicities ranging from $<1 / 100$ th solar to $2.5 \times$ solar. The star formation histories included in our

\footnotetext{
24 The NASA/IPAC Extragalactic Database (NED) is operated by the Jet Propulsion Laboratory, California Institute of Technology, under contract with the National Aeronautics and Space Administration.
}

models (via the "sfhdelayed" module) spanned $0.25-12$ Gyr for the oldest population with e-folding times $0.1-8 \mathrm{Gyr}$. In addition to the stars, we included nebular emission with the default values and reprocessed dust emission using the Dale et al. (2014) model with slopes $\alpha=1-2.5$ and $0 \%$ active galactic nucleus fraction.

The final component of our modeling is the dust attenuation curve, for which we adopted a model based on that of Calzetti et al. (2000) but also includes a "bump" in the UV. The Calzetti et al. (2000) form, although originally derived for starburst galaxies, was later validated by Battisti et al. (2016) for local star-forming galaxies more generally, and Battisti et al. (2017) found evidence for a bump feature in inclined galaxies. We adopt the modification by Buat et al. (2011) and implemented in CIGALE by Buat et al. and Lo Faro et al. (2017) with 35.6 and $217.5 \mathrm{~nm}$ the bump width and wavelength, respectively. The bump amplitude setting is 1.3 , and the slope is modified by -0.38 . We reduce the color excess of old population relative to the young stars by a factor of 0.44 and allow the $E(B-V)$ for the young population to vary between 0.12 and 1.98 .

\subsection{Stellar Mass, SFR, and Rest-frame Color}

Once each galaxy's SED has been fitted, a number of physical parameters as well as the rest-frame absolute magnitudes are then extracted from the resulting stellar population models. Figures 12-14 illustrate the measurements gleaned from SED fitting for the complete CASBaH galaxy database. As shown in Figures 12 and 13, the locus of measured stellar masses spans from $M_{*} \approx 10^{8}-10^{11} M_{\odot}$ with a median $\bar{M}_{*} \approx 10^{10.1} M_{\odot}$. at $\bar{z}=0.28$. These values are driven by our Hectospec sample. The SFRs in Figure 13 show that the majority of our galaxies are star-forming. We have compared our results to measurements for other $z<1$ surveys (e.g., PRIMUS; Moustakas et al. 2013) and find similar results. Lastly, the color-magnitude diagram (Figure 14 reveals the bimodal populations of star-forming and red-and-dead galaxies. In summation, the basic measured properties of galaxies discovered in our survey reproduce/follow the typical trends and distributions characteristic of any other low- $z$ sample. 

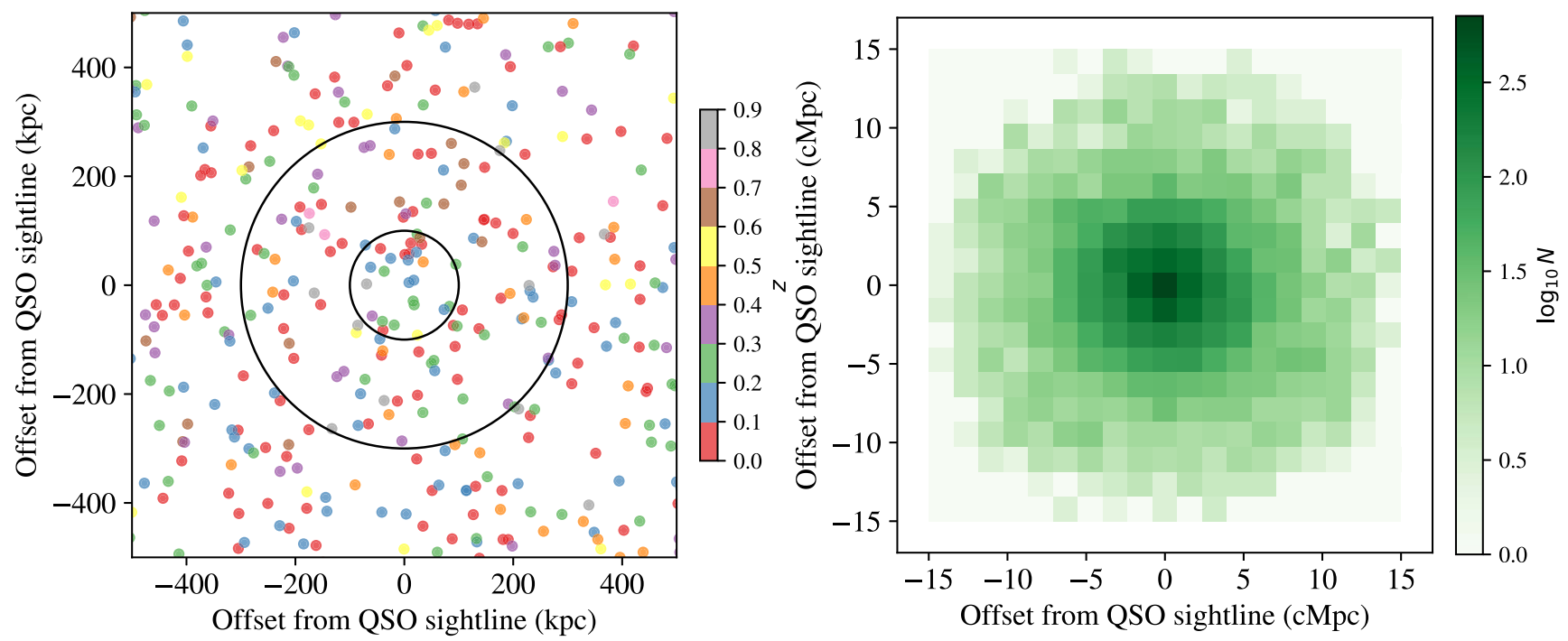

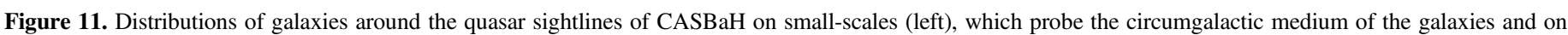
large scales (right), which trace the distribution of the intergalactic medium. Annuli in the left panel indicate impact parameters of 100 and $250 \mathrm{kpc}$.

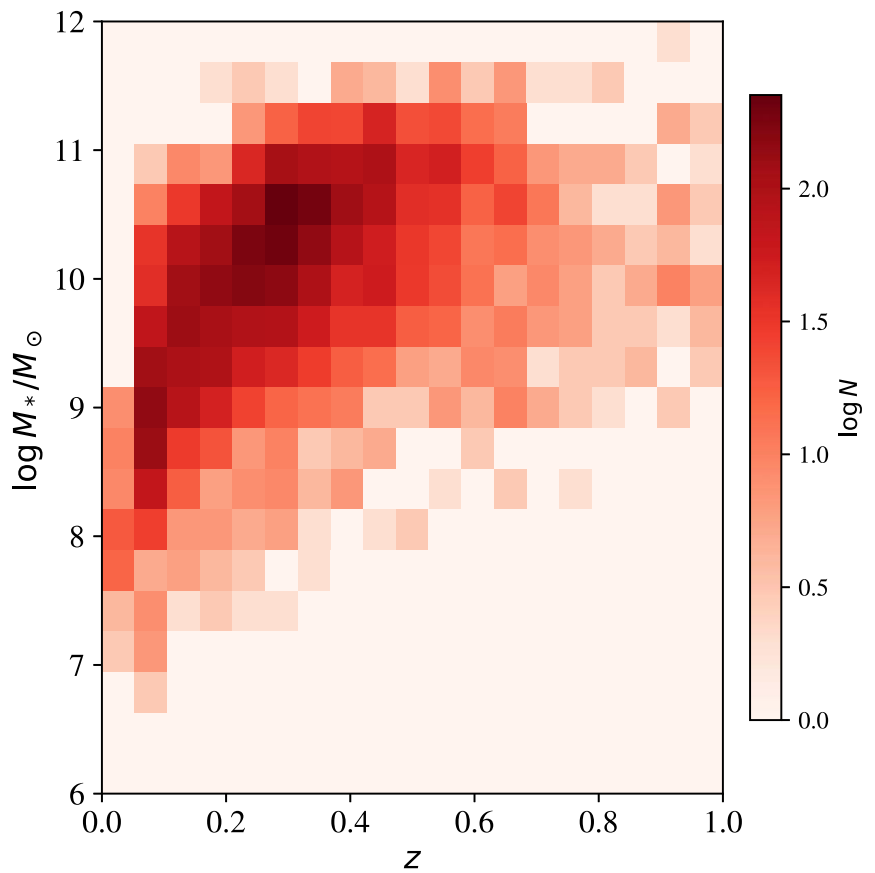

Figure 12. Distribution of stellar mass vs. redshift for the spectroscopically confirmed galaxies comprising the CASBaH sample. The median of the sample occurs at $\bar{z}=0.28$ and $\bar{M}_{*} \approx 10^{10.1} M_{\odot}$.

\section{Identification and Measurement of O VI Absorbers}

In the following section, we will study the study the clustering of $\mathrm{O}$ VI absorbers with galaxies using the $\mathrm{CASBaH}$ database. The full description of the CASBaH UV QSO spectroscopy and data handling is presented in T. Tripp et al. (2019, in preparation); in this section, we summarize aspects of the OVI identifications and measurements that are important for the absorber sample definition and O VI-galaxy clustering analysis.

We identified the O VI absorption lines using the multipass line-identification procedure described by Tripp et al. (2008). In the first pass through the data, we simply searched for lines with the signature of the OVI doublet, i.e., lines with the relative separation and relative strengths of the O VI 1031.926

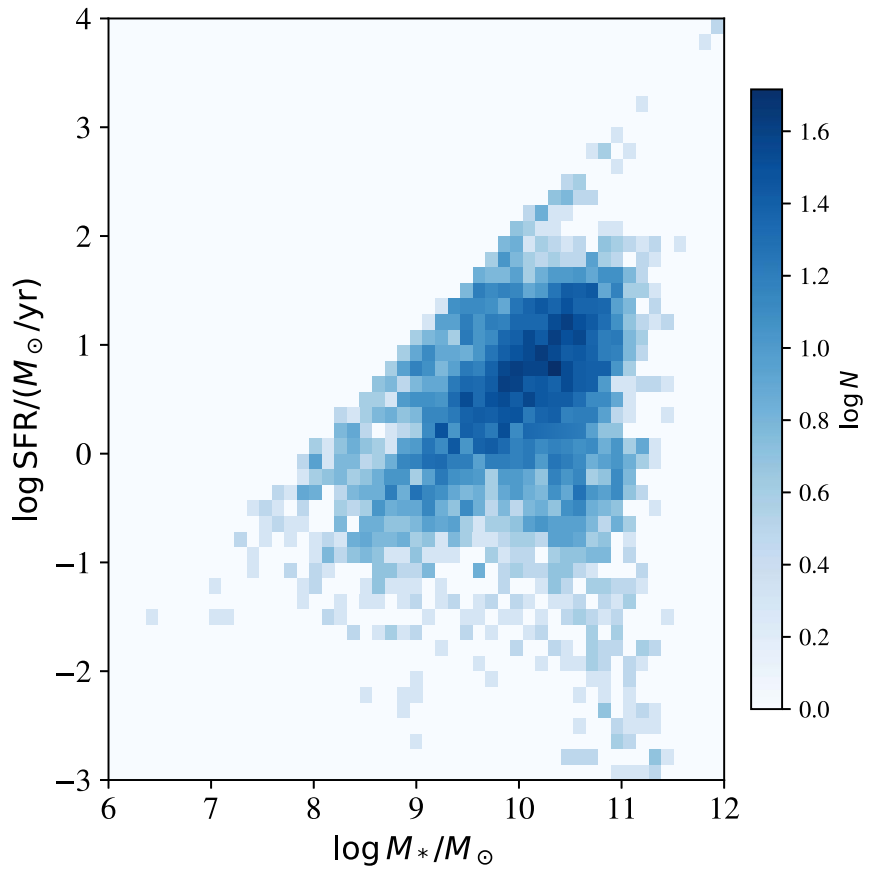

Figure 13. Star formation rate vs. stellar mass from spectral energy density fitting for the CASBaH galaxy sample, where one clearly recognizes the locus of galaxies known as the star-forming main sequence. The hard diagonal edge at the highest SFR for each stellar mass bin occurs due to failures/outliers fitted with models at the boundaries of parameter space.

and $1037.617 \AA$ transitions (see, e.g., Verner et al. 1994). In this pass, we did not require detection of any corresponding $\mathrm{H} \mathrm{I}$ or metal lines; we only searched for the O VI doublet by itself. This first pass identified the majority of the $\mathrm{O}$ VI absorbers, but in some cases, evidence of blending with lines from other redshifts was clearly evident. This is not surprising given the moderately high density of lines in the CASBaH QSO spectra (see Figure 1 in Tripp 2013). In addition, in some cases, one line of the doublet is so severely blended with a strong feature from a different redshift that both lines of the doublet could not be directly recognized in our first pass through the data. To overcome these blending issues, we iteratively made 


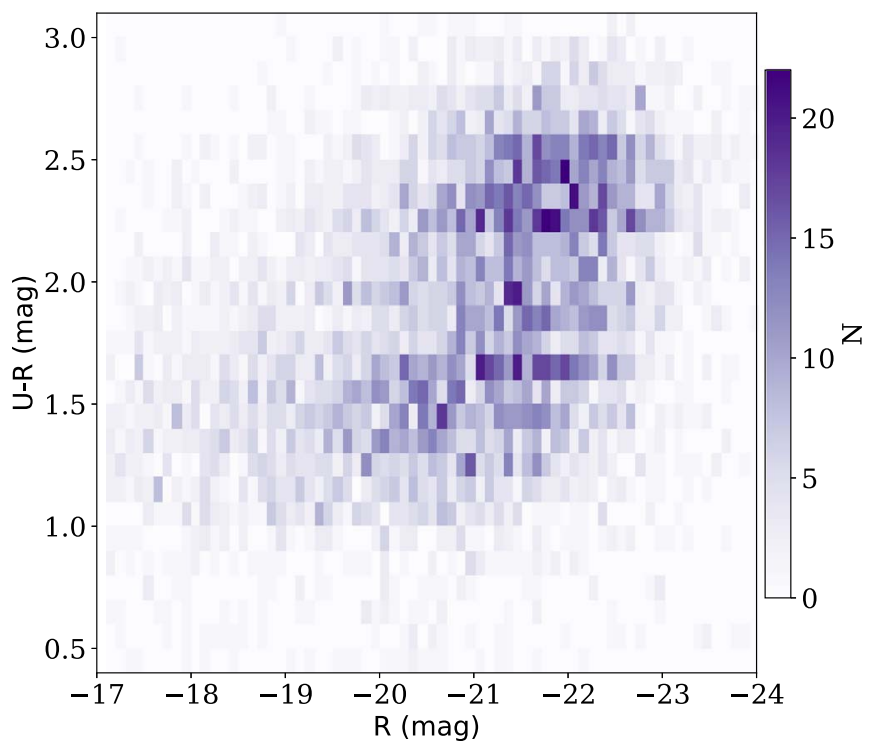

Figure 14. Color-magnitude diagram (rest-frame) for galaxies comprising the CASBaH sample. Evident is the well-known bimodal populations of starforming and "red-and-dead" galaxies.

subsequent passes through the spectra in which we added information about absorption systems established by the presence of HI lines (often with many lines of the Lyman series) and various metals. CASBaH absorbers often show many metal and $\mathrm{H}$ I lines with distinctive component structure (e.g., Ribaudo et al. 2011a; Tripp et al. 2011; Lehner et al. 2013; Meiring et al. 2013), and we used the detailed correspondence of candidate O VI lines with other metals at the same redshift to identify additional O VI absorbers when one or both of the O VI lines was affected by blending (for examples with Ne VIII lines, see Burchett et al. 2018). Since the $\mathrm{CASBaH}$ spectra fully cover the $\mathrm{HI}$ Ly $\alpha$ region from $\lambda_{\mathrm{ob}}=1216$ to $\left(1+z_{\mathrm{QSO}}\right) \times 1216$, we are able to identify nearly all of the lines and systems in the spectra (not just the $\mathrm{O}$ VI systems), including the lines that are blended with the $\mathrm{O}$ VI doublets. Often the interloping lines that are blended with an O VI absorber can be modeled and removed based on lines recorded elsewhere in the CASBaH spectra, and then comparison of the deblended data further corroborates the identification.

In this paper, we are only interested in the foreground/ intervening absorption systems (far from the background QSOs) and their relationships with foreground galaxies. To avoid contaminating our intervening-absorber sample with "proximate" ( $\left.z_{\mathrm{abs}} \approx z_{\mathrm{QSO}}\right)$ absorbers that often comprise material ejected by the QSO that is close to the QSO's central engine (Misawa et al. 2007; Ganguly et al. 2013), we exclude O VI absorbers within $5000 \mathrm{~km} \mathrm{~s}^{-1}$ of the QSO redshift. ${ }^{25}$

All of the intervening O VI absorbers that we identify exhibit $\mathrm{H}$ I absorption at very similar velocities and often show other metal lines (at least C III or O IV, and often other metals); we do not find any unambiguous O VI systems without any

\footnotetext{
${ }^{25}$ In addition, in a few cases we exclude systems at somewhat higher ejection velocities when they exhibit obvious characteristics of "mini-broad absorption line (mini-BAL)" systems such as partial covering, smooth absorption profiles that are much broader than intervening absorption profiles, and strong absorption by exotic and highly ionized species such as $\mathrm{Na}$ IX and $\mathrm{Mg} \mathrm{X}$ (see examples in Muzahid et al. 2013). However, inclusion or exclusion of these mini-BAL systems has no impact on the analysis in this paper because they are all at higher redshifts than our O VI-galaxy clustering sample.
}

corresponding H I. This is consistent with previous studies of low-redshift O VI absorbers, which have shown that the "H Ifree" OVI systems are only found in proximate cases with $z_{\text {abs }} \approx z_{\mathrm{QSO}}$ (Tripp et al. 2008). We note that some intervening $\mathrm{O}$ VI systems have interesting individual $\mathrm{O}$ VI components with corresponding $\mathrm{H}$ I that is very weak (or absent altogether; see, e.g., Tripp et al. 2008; Savage et al. 2010), but these cases are not H I-free; on the contrary, these absorbers have very strong corresponding $\mathrm{H}$ I absorption in some of the components of the overall system. For example, Savage et al. (2010) have analyzed the OVI system at $z=0.167$ in the spectrum of PKS0405-123. This system includes an O VI component at $v=-278 \mathrm{~km} \mathrm{~s}^{-1}$ that is not detected in $\mathrm{HI}$, but the same system also shows $\mathrm{O}$ VI components at $v \approx-125,-50$, and $0 \mathrm{~km} \mathrm{~s}^{-1}$, and these lower-velocity components have strong corresponding H I absorption (see Figure 3 in Savage et al. 2010). Thus, this example has an H I-free component, but it is not an $\mathrm{H}$ I-free system; similar intervening systems are found in the CASBaH database. Only the proximate absorption systems are entirely free of $\mathrm{HI}$ in all of the components (some examples of such proximate systems are shown in Figures 7 and 20 of Tripp et al. 2008).

To measure the absorption-line properties, here we primarily rely on fitting multicomponent Voigt profiles to the data using the software developed by Burchett et al. (2015). These models constrain the redshift, line width, and column density of each O VI component, and we aggregate the components into "systems" as described below. To assess the significance of the candidate lines, we use the Voigt-profile parameters to calculate the equivalent width, which we then compare to the limiting equivalent width (calculated using the method of Tripp et al. 2008) at that wavelength. In this paper we have focused on well-detected lines ( $>4 \sigma$ significance) that are not involved in very complicated blends that preclude robust profile fitting. In this conservative sample, we find a paucity of lines with log $N(\mathrm{O}$ VI $)<13.5$, and we also impose a lower limit on the $\mathrm{O}$ VI column density of absorbers that are included in the clustering analysis (see below).

Figure 15 provides a snapshot of the overall database (galaxy and OVI absorber information) using the PG1407+265 sightline and field as an example. In the top panel of this figure, we show the full galaxy database in a cylinder with radius $=3 \mathrm{Mpc}$ centered on the QSO. The middle panel illustrates the column densities of the OVI lines are their locations with respect to the nearby galaxies and large-scale structures that can be seen in the upper panel, and the lower panel shows the $3 \sigma$ limiting (rest-frame) equivalent width as a function of $\mathrm{O} V I$ redshift with the equivalent widths of the detected O VI lines overplotted. Comparing the O VI and galaxy impact parameters, we see that O VI lines are typically detected when there is a galaxy close to the line of sight, but there is substantial scatter in the O VI column at a given impactparameter value. This is consistent with previous studies (e.g., Stocke et al. 2006; Tumlinson et al. 2011; Johnson et al. 2015). We will present analyses of the connections of individual O VIgalaxy papers in a separate paper (J. Burchett et al. 2019, in preparation). We also see from Figure 15 that the $\mathrm{CASBaH}$ database provides extensive information on galaxy structures on large scales, which is the focus on the galaxy-absorber analyses in this paper. 

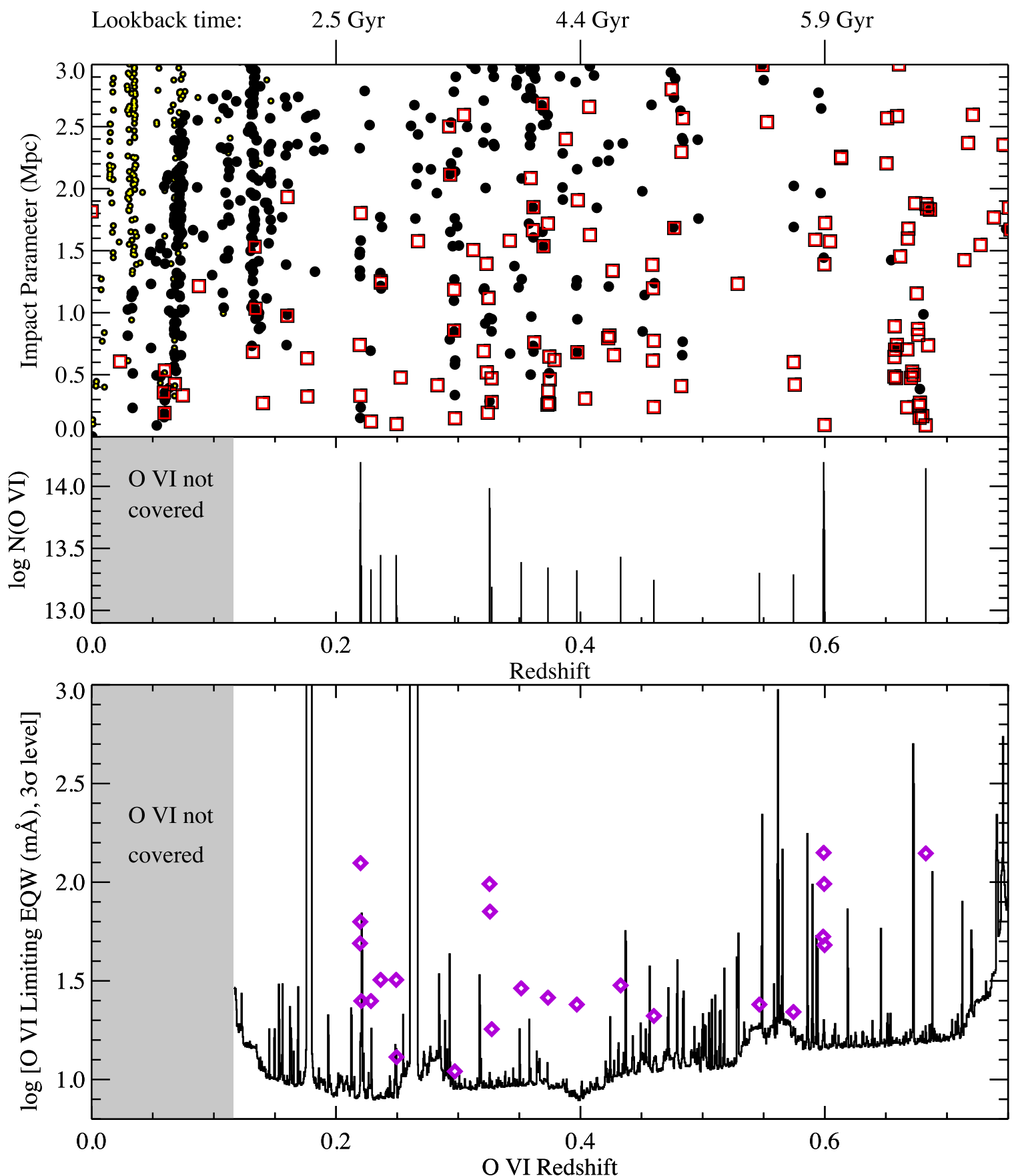

Figure 15. Top: Galaxies in the CASBaH database for PG1407+265 as a function of redshift, within impact parameters of $3 \mathrm{Mpc}$ or less at $z_{\mathrm{gal}}<0.75$. Galaxies with redshifts from the SDSS are indicated by small circles with yellow centers, galaxy redshifts measured with Hectospec are represented with larger filled-black circles, and galaxies measured with DEIMOS are shown with red squares. For reference, lookback times are indicated on the top axis. Middle: logarithmic column densities and redshifts of the O VI absorption lines detected in the UV spectra of PG1407+265. Each vertical line marks the redshift of an O VI component, and the height of the line indicates $\log N(\mathrm{O} \mathrm{VI})$. Bottom: the $3 \sigma$ limiting equivalent width for detection of $\mathrm{O}$ VI in the PG1407+265 data vs. redshift. The equivalent widths of detected $\mathrm{O}$ VI components are plotted as purple diamonds.

\section{O VI-Galaxy Clustering}

With the galaxy spectral database of CASBaH constructed, we proceed to measure the clustering of CASBaH galaxies with themselves (auto-correlation) and with O VI absorption systems (cross-correlation) in the $z<1$ universe. Our scientific motivations are two-fold: (i) to further characterize the galaxy sample of the CASBaH survey, and (ii) to provide new estimates on the masses of galaxies associated with $\mathrm{OVI}$ absorption. Regarding the latter goal, we emphasize that the results derived will only apply for O VI systems with properties similar to those drawn from CASBaH. Furthermore, any estimate on mass follows from the ansatz that the majority of these O VI systems arise within dark matter halos. We also emphasize that incompleteness in the galaxy and O VI samples is accounted for by the estimator and procedures adopted in the clustering analysis. Finally, we restrict the analysis to comoving separations in excess of $1 h_{100}^{-1} \mathrm{Mpc}$ to isolate the so-called two-halo term that results from large-scale clustering. 


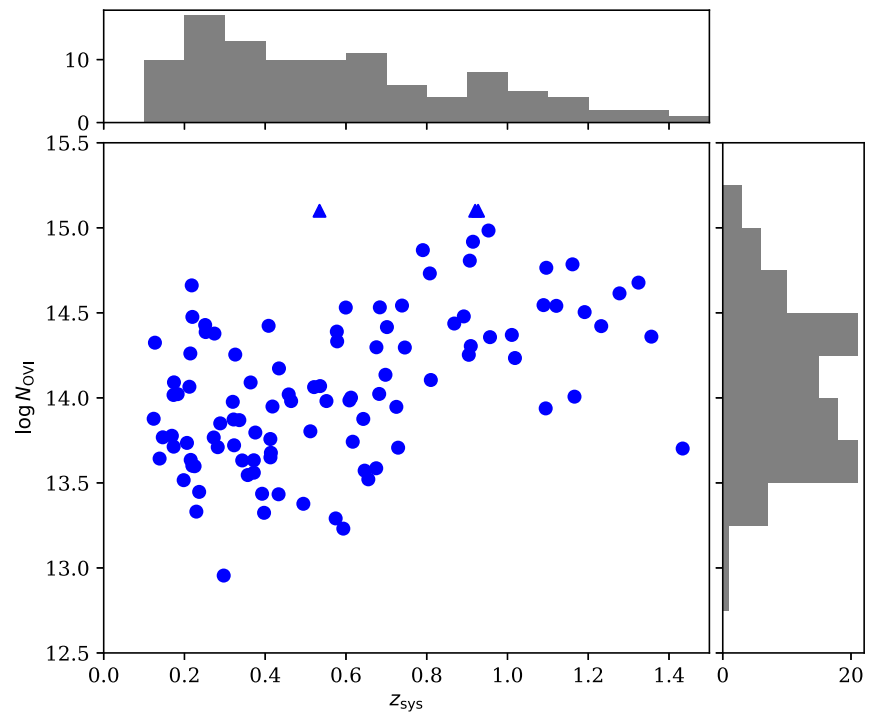

Figure 16. Scatter plot and histograms of column densities vs. redshift for the $\mathrm{O}$ VI systems detected in the CASBaH survey. Typical uncertainties in $N(\mathrm{O}$ VI) are $\approx 0.03$ dex. The measurements with $N(\mathrm{O}$ VI $)>10^{15} \mathrm{~cm}^{-2}$ are saturated, are reported as lower limits, and are designated with triangles. The lack of of weaker O VI absorbers at $z>0.75$ is driven by the shift of the O VI doublet from the FUV to the NUV bandpass where the spectra have lower signal-tonoise levels.

\subsection{Setup}

This sub-section describes cuts on our absorber and galaxy databases used to generate a well-defined set of systems and galaxies for the analysis. To measure O VI-galaxy clustering, we define a discrete set of O VI systems along the sightlines, with each system characterized by a systemic redshift $z_{\text {sys }}$ and column density $N(\mathrm{O}$ VI). To achieve this, our approach in this $\mathrm{CASBaH}$ paper is to synthesize components (provided by $\mathrm{T}$. Tripp et al. 2019, in preparation) into absorption systems. Using the MeanShift clustering algorithm from the scikit-learn Python package (Pedregosa et al. 2011), we grouped components clustered in velocity space into absorption systems, setting the redshift of each system to the center of its component cluster. The MeanShift algorithm accepts a bandwidth argument, which defines an approximate width within which to group components. We chose a bandwidth value of $600 \mathrm{~km} \mathrm{~s}^{-1}$ for component clustering, finding that this value was large enough to produce systems with components spread over a large range of velocity $\left(\sim 1000 \mathrm{~km} \mathrm{~s}^{-1}\right)$, such as the post-starburst outflow analyzed by Tripp et al. (2011), but small enough to separate systems with components clustered about discrete center points with large separations (also on the order of $1000 \mathrm{~km} \mathrm{~s}^{-1}$ ). For systems composed of multiple components, we sum their individual column densities to yield a total $\mathrm{N}(\mathrm{O} \mathrm{VI})$ for each system.

These values are shown in Figure 16 as a function of system redshift. At $z<0.75$, the sample scatters from $N(\mathrm{O}$ VI $) \approx 10^{13.3}-10^{14.5}$ with no discernible redshift evolution (the cut-off at $z \approx 0.12$ is due simply to lowest observed wavelength of our FUV spectra, $\lambda_{\text {ob }}=1152 \AA$ ). At higher redshifts, the $N(\mathrm{O} \mathrm{VI})$ values are systematically higher (see the discussion in T. Tripp et al. 2019, in preparation). These issues motivate sample criterion 1: the clustering analysis is restricted to $0.12<z<0.75$. This criterion is also motivated by the small number of galaxies that we have observed at higher redshift (e.g., Figure 10).

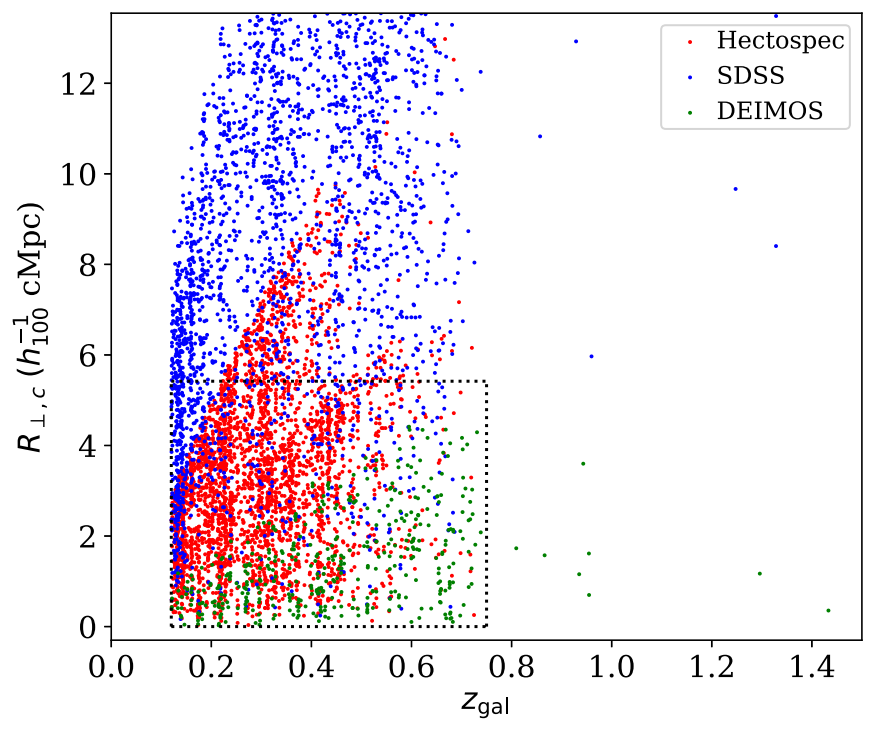

Figure 17. Scatter plot of the comoving impact parameter $R_{\perp, c}$ vs. galaxy redshift for the $\approx 6000$ galaxies with redshifts corresponding to CASBaH absorption data that are sufficiently sensitive to detect an O VI system with $N(\mathrm{O} \mathrm{VI}) \geqslant 10^{13.5} \mathrm{~cm}^{-2}$. The dotted box illustrates the $R_{\perp, c}, z_{\text {gal }}$ parameter space used for our O VI-galaxy cross-correlation analysis.

Further examining the O VI column density histogram, one notes a marked decline in the number of systems with $\log N(\mathrm{O}$ VI $)<13.5$; $\quad$ e.g., only one system exhibits $\log N(\mathrm{O}$ VI $)<13.2$. We emphasize that this dropoff is not entirely driven by sensitivity. Indeed, a substantial fraction of the CASBaH FUV spectra have a $2 \sigma$ limit on $N(\mathrm{O}$ VI) that is lower than $10^{13.5} \mathrm{~cm}^{-2}$ (for O VI $\lambda 1031$ over an integration window of $60 \mathrm{~km} \mathrm{~s}^{-1}$ ). Therefore, the observed distribution implies a physical turn-over in the $N(\mathrm{O} \mathrm{VI})$ frequency distribution as also reported by Danforth et al. (2016); this will be explored further in a future paper. For the clustering study, we are motivated to set a minimum column density threshold $N(\mathrm{O} \mathrm{VI})_{\text {lim }}$ for including $\mathrm{O}$ VI systems in the analysis. This provides a well-defined sample and we can set $N(\mathrm{O}$ VI) to be sufficiently high to include nearly all of the COSFUV data. We find that $N(\mathrm{O} \text { VI })_{\lim }=10^{13.5} \mathrm{~cm}^{-2}$ satisfies this goal and also includes the majority of $\mathrm{O}$ VI systems detected. This motivates sample criterion 2: we restrict the $\mathrm{O}$ VI systems to those with $N(\mathrm{O} \mathrm{VI}) \geqslant N(\mathrm{O} \mathrm{VI})_{\text {lim }}$. The primary exceptions to an approximately uniform sensitivity in the COS-FUV spectra are spectral regions absorbed by other, unrelated systems (e.g., Lyman series absorption by a system at higher redshift). For each galaxy at $0.12<z<0.75$, we have assessed the spectra in a $\pm 30 \mathrm{~km} \mathrm{~s}^{-1}$ window centered at the O VI $\lambda 1031$ wavelength and find that $\approx 16 \%$ have a significant blend that prohibits sensitivity to $N(\mathrm{O} \mathrm{VI})_{\lim }$. This motivates sample criterion 3: we ignore galaxies whose redshift places them in a blend that prohibits measuring down to $N(\mathrm{O} \mathrm{VI})_{\lim }$, despite the overall high $\mathrm{S} / \mathrm{N}$ of the spectra.

Figure 17 illustrates the set of galaxies satisfying the three sample criteria, plotted at their comoving distance from the quasar sightline. To enforce the $N(\mathrm{O} \text { VI })_{\text {lim }}$ criterion, we estimated the uncertainty in column density by integrating the apparent optical depth at O VI $\lambda 1031$ in a window of $\pm 30 \mathrm{~km} \mathrm{~s}^{-1}$ centered on each galaxy redshift. We then flagged those with $2 \sigma(N(\mathrm{O} \mathrm{VI}))<10^{13.5} \mathrm{~cm}^{-2}$, without a blend, and with $z_{\text {gal }}>0.12$. Altogether we have $\approx 6000$ galaxies in the $\mathrm{CASBaH}$ survey satisfying these criteria. It is apparent from 
Figure 17 that very few galaxies with $z_{\text {gal }}>0.75$ have sufficient $\mathrm{S} / \mathrm{N}$ at $\mathrm{O}$ VI to satisfy the sensitivity limit (see also T. Tripp et al. 2019, in preparation). One also notes that, at fixed redshift, the $\mathrm{CASBaH}$ survey has a roughly constant galaxy sampling to $R_{\perp, c} \approx 5.4 \mathrm{~h}_{100}^{-1} \mathrm{cMpc}$ which declines monotonically with increasing $z_{\mathrm{gal}}$. Beyond $R_{\perp, c} \approx 5.4 h_{100}^{-1} \mathrm{Mpc}$, one identifies striping related to the wedding cake design of the Hectospec observations (Section 2.3.1). This leads to sample criterion 4: restrict the cross-correlation analysis to $R_{\perp, c}<5.4 h_{100}^{-1} \mathrm{Mpc}$.

Finally we restrict the analysis to the seven fields observed with Hectospec, DEIMOS, or both (i.e., PG1338+416 and LBQS1435-0134 are not used in this paper). The fields with only SDSS coverage have too few galaxies (i.e., too large shot noise) for a meaningful analysis.

\subsection{Galaxy-Galaxy Auto-correlation}

Interpretation of the results from the O VI-galaxy crosscorrelation analysis will depend on the nature of the galaxies that comprise the CASBaH survey. We have assessed several intrinsic properties in the previous section (e.g., Figure 12); here we perform an auto-correlation analysis to further assess the halo mass of the population.

Our methodology follows closely that of Tejos et al. (2014) who studied the clustering of $\operatorname{Ly} \alpha$ absorption with galaxies. ${ }^{26}$ Their approach compares the incidence of galaxy-galaxy (or galaxy-absorber) pairs at a given comoving separation with the incidence of "random" pairs derived from properties of the survey design. Specifically, they adopt the Landy-Szalay formalism (L-S; Landy \& Szalay 1993). Of particular importance to the analysis is matching the redshift and impact parameter distributions of the random and real samples as a function of apparent magnitude. Regarding the former, Figure 18 compares the observed redshift distributions for galaxies discovered with Hectospec (outer layer, $\theta>10^{\prime}$; see Section 2.3.1) as a function of $r$-band magnitude against a random distribution drawn from a cubic-spline representation fit to a Gaussian-smoothed histogram of the real distributions. We refer to these cubic-splines as sensitivity functions because they depend on the magnitude limit of the galaxies targeted and the quality of the spectroscopy. Importantly, the sensitivity functions are designed to smooth out redshift "spikes" in the real observations while maintaining the general distribution. Similar sensitivity functions were derived from each subset of the spectroscopic survey (i.e., SDSS, DEIMOS, other Hectospec layers), also in cuts of galaxy magnitude. The agreement between data and randoms is shown in Figure 18 for the Hectospec (outer layer) subset. The sensitivity functions were derived by combining data from all of the fields with the exception of the PG1630+377 field. We found it necessary to generate a custom sensitivity function for the Hectospec outer subset due to a large overdensity at $z \approx 0.4$ in that field.

For each real galaxy, a set of $n_{\text {rand }}=100$ galaxies were placed at its R.A./decl. with redshifts drawn randomly from the appropriate sensitivity function. Figure 19 compares the distribution of comoving separations of the real and random galaxies for the sightlines. The close correspondence is vital to the analysis. For each field, we then evaluate the number of data-data $\left(D_{g} D_{g}\right)$ data-random $\left(D_{g} R_{g}\right)$ and random-random

\footnotetext{
${ }^{26}$ All of the code is available in the PYIGM repository on GitHub (https:// github.com/pyigm/pyigm).
}
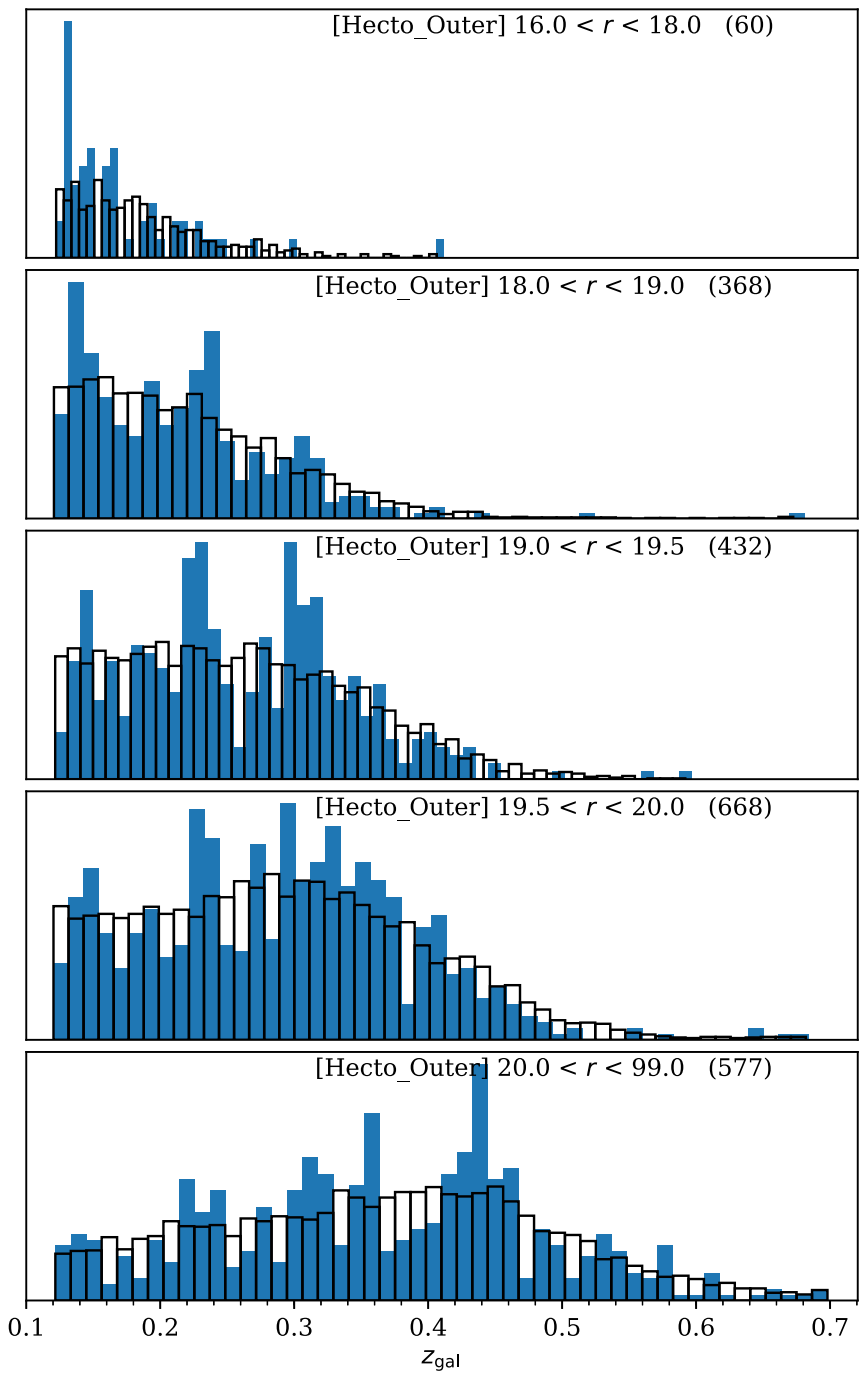

Figure 18. Blue, filled histograms show the redshift distribution for galaxies observed in the outer layer of the Hectospec data set $\left(\theta>10^{\prime}\right)$, split by $r$-band magnitude. The number in parenthesis lists the total in each interval. Overlaid in the open, black histogram is a normalized, random distribution drawn from a cubic-spline fit to a Gaussian-smoothed version of the real histogram. This smooths out the small-scale clustering of the galaxies while maintaining the overall distribution.

$\left(R_{g} R_{g}\right)$ galaxy-galaxy pairs in bins of $0.339 h_{100}^{-1} \mathrm{Mpc}$ in the radial $\left(R_{\|}\right.$, line of sight) and tangential ( $R_{\perp}$, plane-of-sky) directions. We then sum all of the fields and use the L-S estimator:

$$
\xi_{\mathrm{gg}}(r)^{L S}=\frac{D_{g} D_{g} / n_{\mathrm{gg}}^{D D}-2 D_{g} R_{g} / n_{\mathrm{gg}}^{D R}}{R_{g} R_{g} / n_{\mathrm{gg}}^{R R}}+1
$$

to evaluate $\xi_{\mathrm{gg}}(r)$ with $n_{\mathrm{gg}}^{D D}, n_{\mathrm{gg}}^{D R}$, and $n_{\mathrm{gg}}^{R R}$, the normalization factors. Figure 20 shows the binned evaluation.

Uncertainties in $\xi_{\mathrm{gg}}(r)$ have been estimated from the analytic approximation of the variance presented by Landy \& Szalay (1993) as (in our notation)

$$
\sigma_{\xi_{\mathrm{gg}}}^{2}(r)^{L S} \approx \frac{\left(1+\xi_{\mathrm{gg}}^{L S}\right)^{2}}{n_{\mathrm{gg}}^{D D}\left(R_{g} R_{g} / n_{\mathrm{gg}}^{R R}\right)} \approx \frac{\left(1+\xi_{\mathrm{gg}}^{L S}\right)^{3}}{D_{g} D_{g}} .
$$

As typical of such analysis, one observes an asymmetry due to peculiar motions in converting redshift into distance along the 


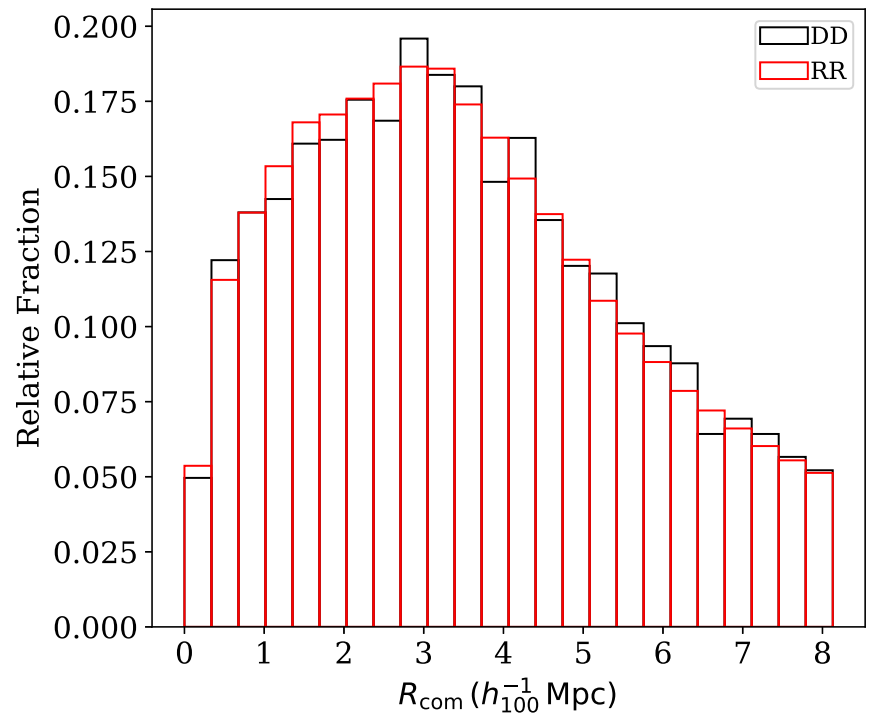

Figure 19. Normalized histograms comparing the distribution of projected separations from the quasar sightline for the real galaxy sample (DD; black) and the constructed set of random galaxies (RR; red). The close agreement is critical to an unbiased estimate of $\xi_{\mathrm{gg}}(r)$.
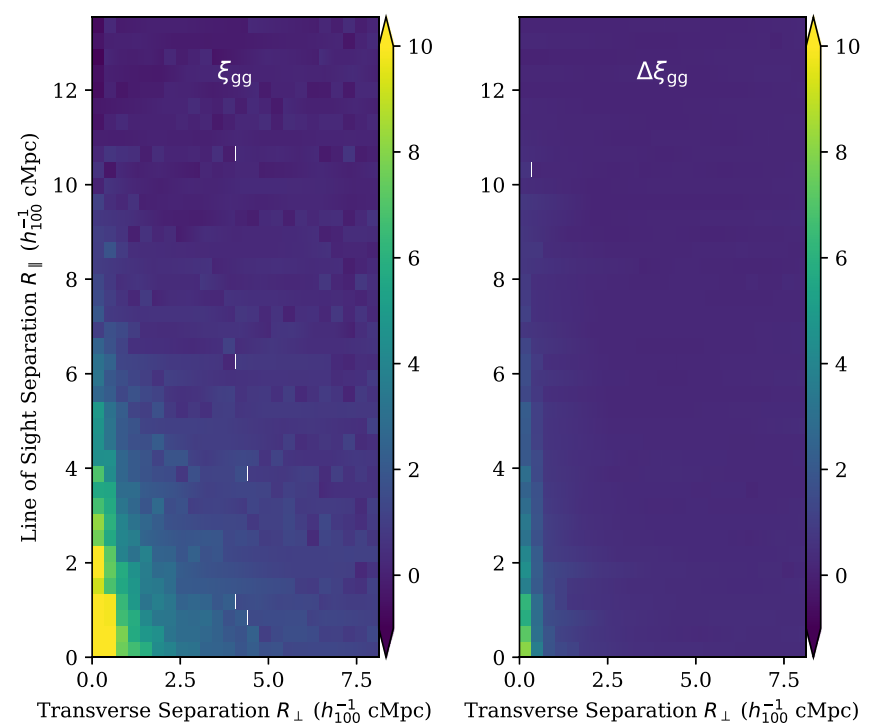

Figure 20. (left) Binned evaluations of the galaxy-galaxy auto-correlation function $\xi_{\mathrm{gg}}(r)$ measured from $z<0.75$ galaxies in the CASBaH survey $\left(0.359 h_{100}^{-1} \mathrm{Mpc}\right.$ grid). (right) Estimates of the uncertainty in $\xi_{\mathrm{gg}}(r)$ derived from the variance of the Landy-Szalay estimator (Equation (2)).

line of sight (i.e., redshift distortions). Examining the measurements in the transverse separation $R_{\perp}$, one also identifies significant $\xi_{\mathrm{gg}}(r)$ signal at large separations which we now quantify.

To reduce the effects of redshift distortion and also to parameterize the $\xi_{\mathrm{gg}}(r)$ measurements, we have evaluated the mean transverse correlation function by averaging $\xi_{\mathrm{gg}}(r)$ along the line of sight to $R_{\|}=13.55 h_{100}^{-1} \mathrm{Mpc}$,

$$
\left\langle\xi_{\mathrm{gg}}^{\mathrm{T}}\left(R_{\perp}\right)\right\rangle=\frac{1}{N} \sum_{i}^{N} \xi_{\mathrm{gg}}^{i}\left(r=R_{\perp}\right),
$$

where the sum is over the 40 bins of $0.339 h_{100}^{-1} \mathrm{Mpc}$ in the line of sight dimension. This quantity is presented in Figure 21,

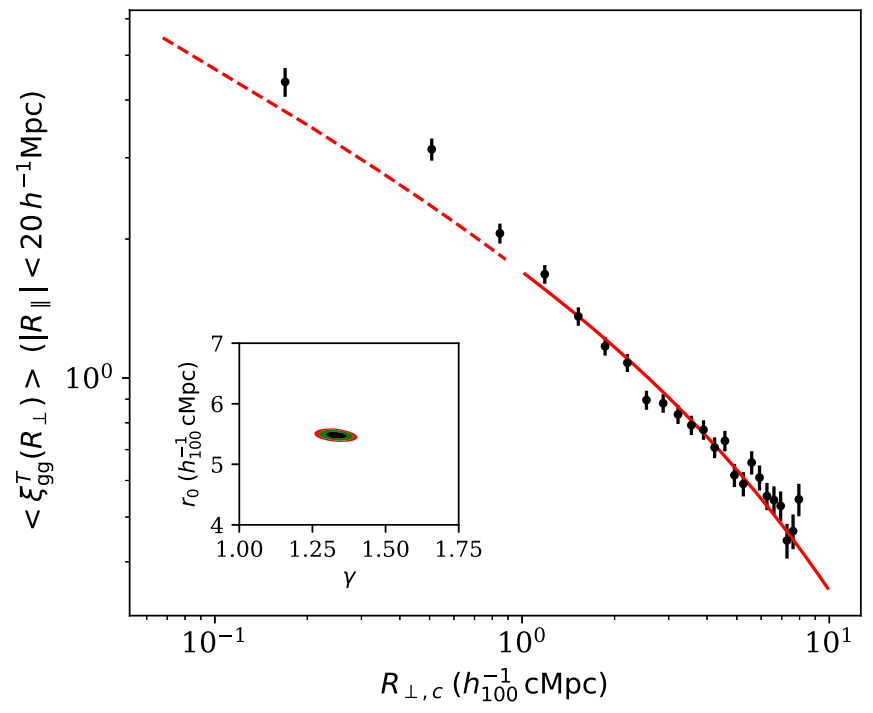

Figure 21. Evaluations of the galaxy-galaxy correlation function averaged along the line of sight to $R_{\|}=13.55 h_{100}^{-1} \mathrm{Mpc},\left\langle\xi_{\mathrm{gg}}^{\mathrm{T}}\left(R_{\perp}\right)\right\rangle$, in bins of transverse separation. The solid red curve shows the evaluation of $\left\langle\xi_{\mathrm{gg}}^{\mathrm{T}}\left(R_{\perp}\right)\right\rangle$ for the best-fit 3D power law for $\xi_{\mathrm{gg}}(r)$ to the data over $R_{\perp, c}=[1-10] h_{100}^{-1} \mathrm{Mpc}$. The dashed curve is an extrapolation of this model to $R_{\perp, c}<1 h_{100}^{-1} \mathrm{Mpc}$ where one notes the data significantly exceed the evaluation. This offset is attributed to galaxygalaxy clustering within dark matter halos, i.e., the one-halo term. The inset shows the confidence contours from a maximum likelihood analysis which yields $r_{0}=5.48 \pm 0.07 h_{100}^{-1} \mathrm{Mpc} h_{100}^{-1} \mathrm{Mpc}$ and $\gamma=1.33 \pm 0.04$ at $68 \%$ c.l.

with uncertainties derived from the L-S estimator (see Equation (2)) for the collapsed pair-counts. Overlaid on the data is the best-fit power-law model for the 3D correlation function $\xi_{\mathrm{gg}}(r)=\left(r / r_{0}\right)^{-\gamma}$ estimated from standard maximum likelihood techniques assuming a Gaussian deviate. The best-fit values are $r_{0}=5.48 \pm 0.07 h_{100}^{-1} \mathrm{Mpc} h_{100}^{-1} \mathrm{Mpc} \quad$ and $\gamma=1.33 \pm 0.04$ with uncertainties referring to $68 \%$ confidence intervals. Specifically, we averaged $\xi_{\mathrm{gg}}(r)$ over $R_{\|}=[0,13.55] h_{100}^{-1} \mathrm{Mpc}$ at the center of each $\left\langle\xi_{\mathrm{gg}}^{\mathrm{T}}\left(R_{\perp}\right)\right\rangle$ bin and constructed the resultant likelihood function by varying $\gamma$ and $r_{0}$. The likelihood evaluation is limited to the transverse bins in the interval $R_{\perp, c}=[1-10] h_{100}^{-1} \mathrm{Mpc}$ to isolate the socalled two-halo term of large-scale galaxy-galaxy clustering. The best-fit correlation length is typical of star-forming galaxies at $z \sim 0.3$ (Coil et al. 2017), which is consistent with the properties of our sample (Figure 12, J. Burchett et al. 2019, in preparation) We also note that the reported uncertainties are likely underestimated because we have not included all sources of variance, e.g., field-to-field variations (Tejos et al. 2014).

The dashed line in Figure 21 is an extrapolation of the $\left\langle\xi_{\mathrm{gg}}^{\mathrm{T}}\left(R_{\perp}\right)\right\rangle$ evaluation to $R_{\perp, c}<1 h_{100}^{-1} \mathrm{Mpc}$. At these separations, the measurements well exceed the model which is generally interpreted as galaxy-galaxy clustering within individual halos, known as the one-halo term. We have also examined $\left\langle\xi_{\mathrm{gg}}^{\mathrm{T}}\left(R_{\perp}\right)\right\rangle$ for sub-samples of the full galaxy data set. Splitting the sample into two redshift bins at $z_{\text {gal }}=0.45$, we estimate $\left\langle\xi_{\mathrm{gg}}^{\mathrm{T}}\left(R_{\perp}\right)\right\rangle$ values that are approximately two times higher for the higherredshift galaxies. This follows from the fact that they are intrinsically more luminous and have higher average stellar mass. Furthermore, the high- $z$ set includes many LRGs from the SDSS which have a very high clustering amplitude (e.g., Nuza et al. 2013). 


\subsection{O VI-Galaxy Clustering}

Inherent to an absorber-galaxy cross-correlation analysis is the notion that absorption systems are measured to occur more (or less) frequently in the proximity of a galaxy than at random. For absorption systems, one can assess the random incidence by surveying many sightlines to estimate the average number per redshift interval, ${ }^{27} \ell_{\mathrm{O} \text { VI }}(z) d z$. Blind surveys for $\mathrm{O}$ VI systems along low- $z$ sightlines have yielded direct estimates of $\ell_{\mathrm{O} \text { vI }}(z)$. Tripp et al. (2008) report $\ell_{\mathrm{O} \text { VI }}(z)=15.6_{-2.4}^{+2.9}$ at $z=[0.1,0.5]$ from a sample of 51 systems along 16 sightlines for an equivalent width limit of $30 \mathrm{m \AA}$ (i.e., about $\log N(\mathrm{O}$ VI $) \simeq 13.3$ ). Danforth et al. (2016) have extended the analysis to 82 sightlines at $z_{\mathrm{OSO}}<0.85$ and we estimate $\ell_{\mathrm{OVI}}(z) \approx 17$ for $N(\mathrm{O} \mathrm{VI})>N(\mathrm{O} \mathrm{VI})_{\lim }=10^{13.5} \mathrm{~cm}^{-2}$ from their reported statistics (their Table 5; redshift path $\Delta z_{\mathrm{OVI}} \approx 14.5$ ). One of the future goals of the CASBaH survey is to measure $\ell_{\mathrm{O} \text { vI }}(z)$ from our sightlines to $z \sim 1$ ( $\mathrm{T}$. Tripp et al. 2019, in preparation). As a first estimate, we report 59 systems with $N(\mathrm{O} \mathrm{VI})>N(\mathrm{O} \mathrm{VI})_{\lim }$ over seven sightlines giving a redshift path of $\Delta z \approx 7(0.75-0.12) \simeq 4$.4. Therefore, we estimate $\ell_{\mathrm{OVI}}(z) \approx 13.5$, consistent with the previous literature (this preliminary estimate is lower because our redshift path is overestimated as it does not take into account parts of the spectra that can be blocked to the $\mathrm{O}$ VI absorption). In the following, we adopt $\ell_{\mathrm{OVI}}(z)=13.5$ at $z=0.2$ and assume that $\ell(X)$ is constant throughout our analysis window.

Before assessing the O VI-galaxy cross-correlation function $\xi_{\mathrm{ag}}(r)$, we begin with an estimate of the covering fraction $f_{C}$ of $\mathrm{O}$ VI gas around $z<1$ galaxies. To associate $\mathrm{O}$ VI with an individual galaxy, one must adopt a redshift (or velocity) window. Previous work on the CGM has found that the majority of associated gas occurs within a few hundred $\mathrm{km} \mathrm{s}^{-1}$ of the galaxy redshift (e.g., Prochaska et al. 2011b; Werk et al. 2013). This also holds for CASBaH (Burchett et al. 2018). In the following, we adopt a window of $\delta v= \pm 400 \mathrm{~km} \mathrm{~s}^{-1}$. We further note that this window is small enough that a chance association with $\mathrm{O} V \mathrm{VI}$ is relatively low. Taking $\ell_{\mathrm{OVI}}(z)$ from above, this implies an average of $\mathcal{N}_{\mathrm{O} \text { VI }}=\ell(z) \delta z \approx 0.03$ systems for $\delta z=(\delta v / c) /(1+z)$.

In a series of arbitrary bins of physical impact parameter $R_{\perp, p}$, we have assessed the fraction of CASBaH galaxies with one or more O VI systems ${ }^{28}$ occurring within $\pm 400 \mathrm{~km} \mathrm{~s}^{-1}$. Any number of galaxies may be associated with a given $\mathrm{O}$ VI system. Figure 22 shows the incidence (or covering fraction, $f_{C}$ ) with uncertainties derived from binomial counting statistics. At small impact parameters $\left(R_{\perp, p}<100 \mathrm{kpc}\right)$, the incidence is very high: $f_{C} \approx 75 \%$. This excess is generally attributed to gas within galaxy halos, i.e., the CGM (see J. Burchett et al. 2019, in preparation, for analysis of the O VI CGM in CASBaH). The covering fraction declines monotonically with $R_{\perp, p}$, as expected, but remains $\approx 2 \times$ higher than random expectation at the largest offsets probed by CASBaH $(\approx 8 \mathrm{pMpc})$. This implies significant O VI-galaxy clustering on these scales, which we now assess.

\footnotetext{
${ }^{27}$ It is also common to evaluate $\ell(X) d X$ with $X$ defined to give a constant incidence if the physical cross-section and comoving number density of the population is constant in time (Bahcall \& Peebles 1969).

${ }_{28}$ As noted in Section 6.1, we ignore galaxies with significant blends at the expected location of O VI in the COS-FUV spectra.
}

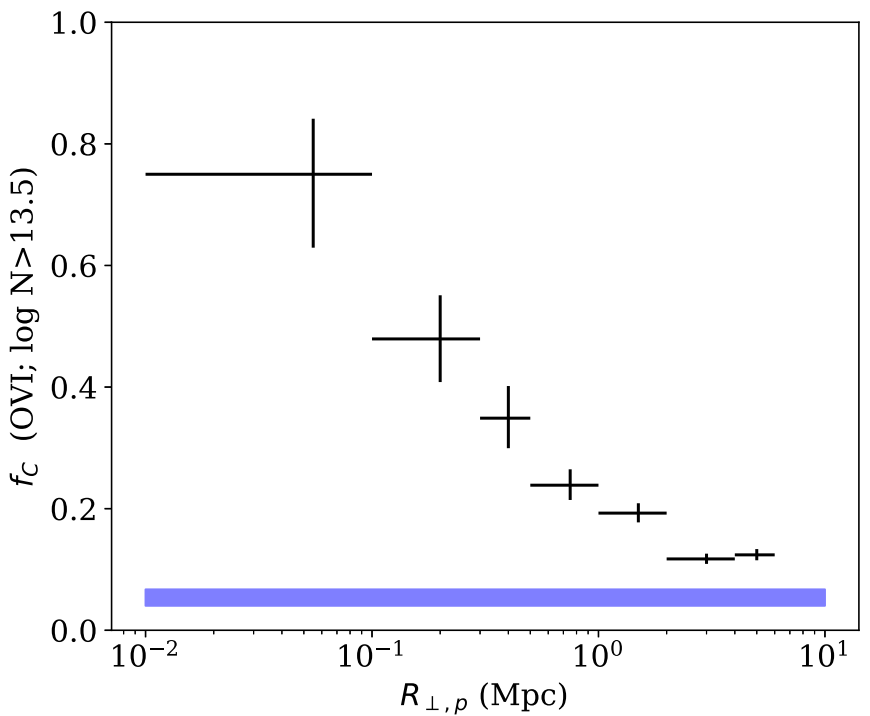

Figure 22. Covering fraction of O VI gas vs. physical impact parameter for galaxies in the $\mathrm{CASBaH}$ survey to a sensitivity limit of $N(\mathrm{O} \mathrm{VI})_{\lim }=10^{13.5} \mathrm{~cm}^{-2}$ with a redshift coincidence within $\pm 400 \mathrm{~km} \mathrm{~s}^{-1}$. There is a high incidence on small scales that may be attributed to gas within galactic halos (i..e the circumgalactic medium). At larger impact parameters $\left(>1 \mathrm{Mpc}\right.$ ), one still recovers $f_{C}$ in excess of random expectation (blue band), indicating significant $\mathrm{O}$ VI-galaxy clustering.

For the cross-correlation analysis of O VI-galaxy clustering, we adopt two approaches. The first follows the analysis developed ${ }^{29}$ by Hennawi \& Prochaska (2007) to evaluate the clustering of optically thick gas around luminous, $z \sim 2$ quasars (see also Prochaska et al. 2013). This analysis uses a maximum-likelihood approach to estimate the 3D crosscorrelation function $\xi_{\mathrm{ag}}(r)$ with an assumed functional form of $\xi_{\text {ag }}(r)=\left(r / r_{0}\right)^{-\gamma}$. The likelihood function is given by $\mathcal{L}=\left(\Pi_{i} P_{i}^{\text {hit }}\right)\left(\Pi_{j} P_{j}^{\text {miss }}\right)$ with $P^{\text {hit }}$ and $P^{\text {miss }}$ the probability of observing one (or more) O VI systems or none, respectively. A galaxy is considered a "hit" if one or more O VI systems occurs within $\pm 400 \mathrm{~km} \mathrm{~s}^{-1}$ and a miss otherwise, and the likelihood is evaluated from the full data set satisfying the sample criteria (Section 6.1). The probability of zero absorbers within a velocity window of $\delta v=400 \mathrm{~km} \mathrm{~s}^{-1}$ is given by Poisson statistics,

$$
P^{\text {miss }}=\exp \left(-\left[1+\chi_{\perp}\right] \ell_{\mathrm{O} \text { vI }}\left(z_{\text {gal }}\right) \delta z\right),
$$

where $\ell_{\mathrm{O} v \mathrm{~V}}(z) d z$ is the mean incidence and $\chi_{\perp}$ expresses the boost from clustering i.e., $1+\chi_{\perp}$, with

$$
\chi_{\perp} \approx \frac{a H(z)}{2 \delta v} \int_{-\delta \mathrm{v} /[\mathrm{aH}(\mathrm{z})]}^{\delta v /[a H(z)]} d R_{\|} \xi_{\mathrm{ag}}\left(\sqrt{R_{\perp}^{2}+R_{\|}^{2}}\right) .
$$

It follows trivially that $P^{\text {hit }}=1-P^{\text {miss }}$.

We constructed a grid of $\mathcal{L}$ by varying $r_{0}$ and $\gamma$ over a range of values and then found the maximum. Figure 23 presents the constraints on $\gamma$ and $r_{0}$ for the subset of CASBaH galaxies analyzed: galaxies

with $R_{\perp, c}=[1,8] h_{100}^{-1} \mathrm{Mpc}, z=[0.12,0.75]$ and also having no substantial blend at the expected location of O VI. We have estimated the uncertainty by integrating $\mathcal{L}$ down to several confidence limits. The best-fit model is shown on a binned evaluation of $\chi_{\perp}$ in Figure 24. This model provides a good

\footnotetext{
${ }^{29}$ Note that we have also corrected their approximation for the probability estimate to be truly Poisson.
} 


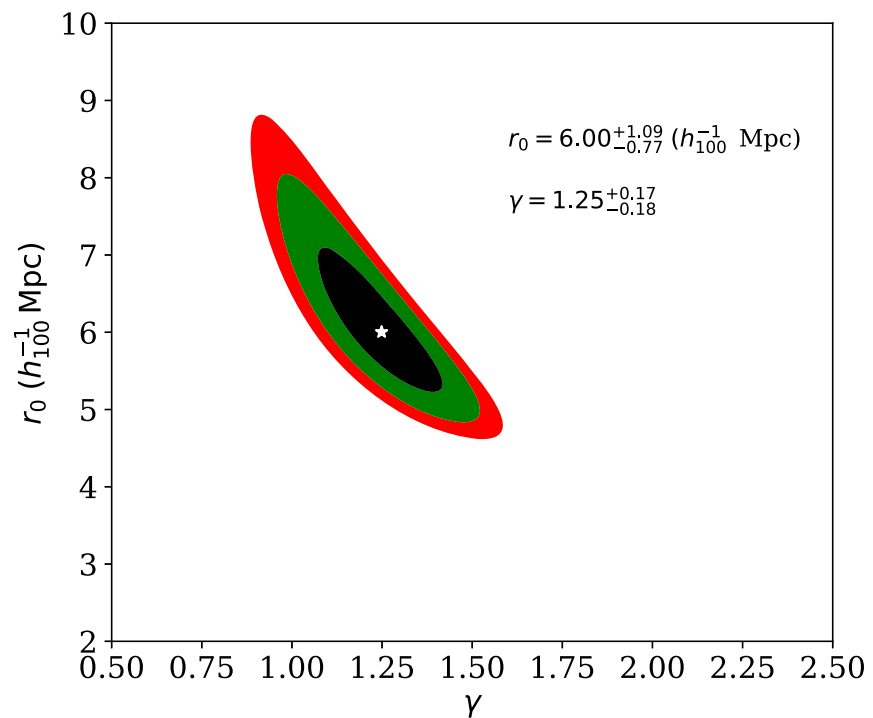

Figure 23. Constraints on $r_{0}$ and $\gamma$ from the $\mathrm{O}$ VI-galaxy cross-correlation analysis, resticted to galaxies with $R_{\perp, c}=[1,8] h_{100}^{-1} \mathrm{Mpc}, z=[0.12,0.75]$ and those without a significant line-blend at O VI.

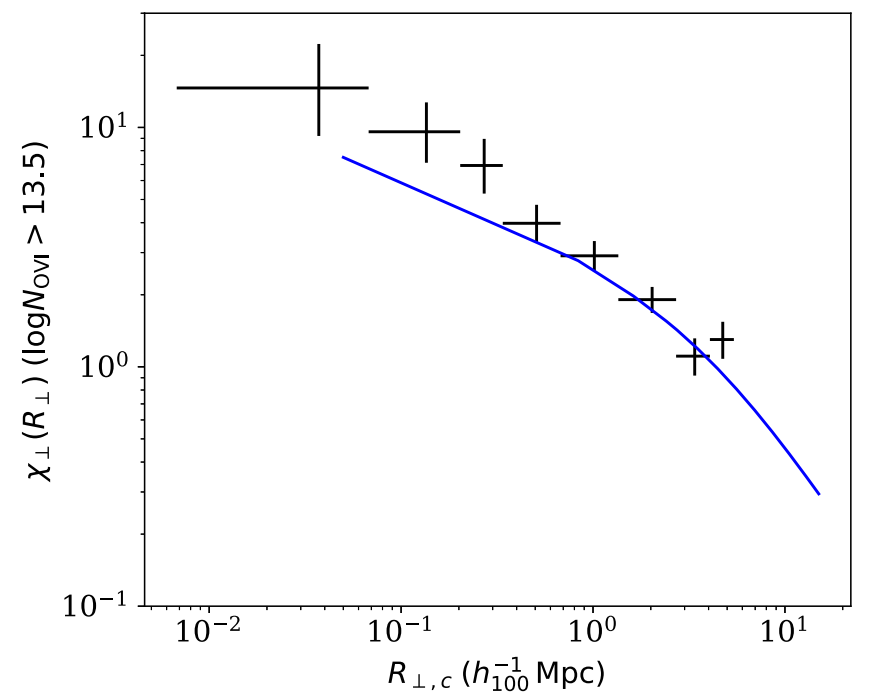

Figure 24. Binned evaluations of $\chi_{\perp}$ derived from the ratio of galaxies with associated $\mathrm{O}$ VI absorption relative to random expectation. Overplotted is the evaluation of Equation (5) using the best-fit parameters derived from a maximum-likelihood analysis (Figure 23).

description of the observations at large values and, as with the galaxy-galaxy clustering, we identify a putative one-halo term at $R<0.5 h_{100}^{-1} \mathrm{Mpc}$, seen as an excess $\chi_{\lrcorner}$over the best fit to larger $R_{\perp}$. We note that our results contrast with the O VIgalaxy clustering study by Finn et al. (2016), who find that the O VI-galaxy signal is lower than the galaxy-galaxy autocorrelation on all scales, while here we find similar correlation lengths between them. Still, given the somewhat shallower slope for the $\mathrm{O}$ VI-galaxy $(\gamma \approx 1.25)$ compared to the galaxygalaxy one $(\gamma \approx 1.33)$, we find that $\xi_{\text {ag }} / \xi_{\text {gg }}$ should be $\lesssim 1$ on scales $\lesssim 1.3 h_{100}^{-1} \mathrm{Mpc}$ (see below). As deeper and more extensive galaxy surveys are completed in the future, it will be valuable to revisit this topic.

We have generated a separate estimate of $\xi_{\mathrm{ag}}(r)$ by analyzing the absorber-galaxy pair counts using the formalism applied to $\xi_{\mathrm{gg}}(r)$ in Section 6.2. In addition to constructing a set of
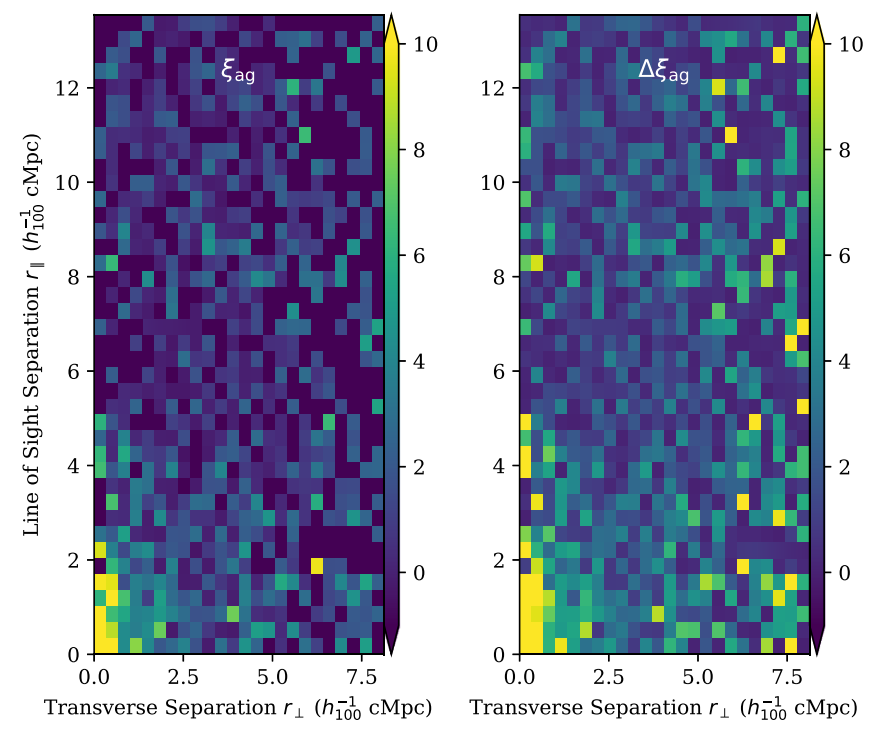

Figure 25. Binned evaluations of $\xi_{\mathrm{ag}}(r)$ and its uncertainty as a function of transverse and line-of-sight separation. Similar to the galaxy-galaxy autocorrelation, we observe the effects of redshift distortions and also detect a significant signal to large separations in $R_{\perp}$.

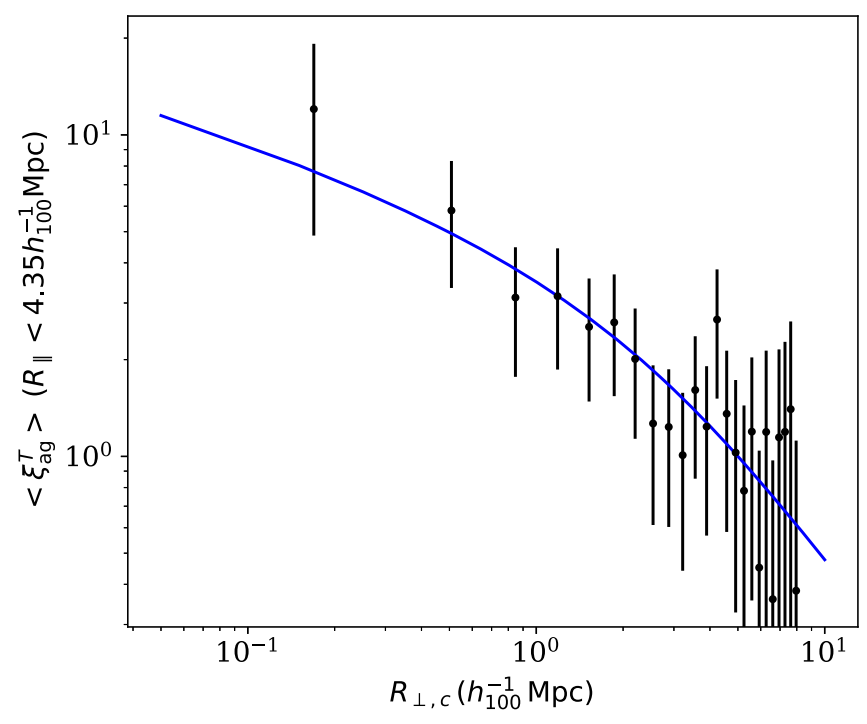

Figure 26. Evaluation of the transverse O VI-galaxy cross-correlation function averaged to $R_{\|}=4.4 h_{100}^{-1} \mathrm{Mpc}$ (corresponding to $\approx 400 \mathrm{~km} \mathrm{~s}^{-1}$ ) from the pair analysis. Measurements and uncertainties were derived from the Landy-Szalay estimator. Overplotted on these values is an evaluation of $\left\langle\xi_{\mathrm{ag}}^{\mathrm{T}}\left(R_{\perp}\right)\right\rangle$ using the best-fit model for $\xi_{\mathrm{ag}}(r)$ from the $\chi_{\perp}$ analysis.

random galaxies, we must also introduce random absorbers for the absorber-galaxy analysis. These were placed along the sightlines with a uniform redshift distribution in the interval $z=[0.12,0.75]$, avoiding strong Galactic ISM absorption (e.g., Si II 1526) which would preclude the detection of O VI.

Figure 25 shows the binned evaluations of $\xi_{\mathrm{ag}}(r)$ and its uncertainty. Similar to the galaxy-galaxy auto-correlation, we observe the effects of redshift distortions and also detect a significant signal to large separations in $R_{\perp}$.

To compare with the $\chi_{\perp}$ analysis from above, we calculate $\left\langle\xi_{\text {ag }}^{\mathrm{T}}\left(R_{\perp}\right)\right\rangle$ by averaging to $R_{\|}=4.4 h_{100}^{-1} \mathrm{Mpc}$ which corresponds to approximately $400 \mathrm{~km} \mathrm{~s}^{-1}$ at $z=0.3$. These estimates are shown in Figure 26 and we also overplot the estimate for $\left\langle\xi_{\text {ag }}^{\mathrm{T}}\left(R_{\perp}\right)\right\rangle$ based on the best-fit model from Figure 23. There is 

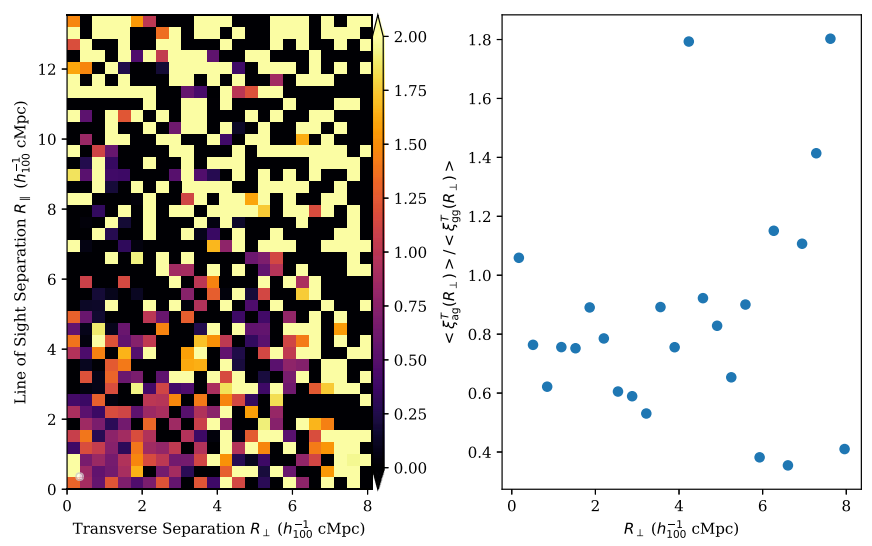

Figure 27. Left: binned evaluation of the ratio $\xi_{\mathrm{ag}}(r) / \xi_{\mathrm{gg}}(r)$ which leads to the evaluation of the bias factor for O VI as given by Equation (6). Right: the same ratio averaged along the line of sight to $4.4 h_{100}^{-1} \mathrm{Mpc}$. From these values we estimate $\xi_{\mathrm{ag}}(r) / \xi_{\mathrm{gg}}(r)=0.76 \pm 0.1$.

good overall agreement between the two techniques, although the pair-counting analysis does yield an $\approx 20 \%$ lower amplitude at most scales. In the following, we use the pair analysis $\xi_{\mathrm{ag}}(r)$ to compare with the auto-correlation function $\xi_{\mathrm{gg}}(r)$ but use the power-law fit to $\chi_{\perp}$ for any further discussion of $r_{0}, \gamma$.

\subsection{Discussion}

We now synthesize the results of the previous sub-sections to derive new insight on the physical association of $\mathrm{O} \mathrm{VI}$ absorption to galaxies. We first remind the reader that the analysis was restricted to $\mathrm{O}$ VI systems with $N(\mathrm{O} \mathrm{VI}) \geqslant 10^{13.5} \mathrm{~cm}^{-2}$ and redshift $0.12<z<0.75$. Furthermore, the galaxy sample is dominated by the Hectospec observations and these have $z \sim 0.2-0.4$ and stellar mass of a few $10^{10} M_{\odot}$ (Figure 12 ).

In the regime of linear bias, we may relate the ratio of the correlation functions to their bias factors,

$$
b_{\mathrm{O} \mathrm{vI}}=b_{\mathrm{gg}} \frac{\xi_{\mathrm{ag}}}{\xi_{\mathrm{gg}}},
$$

and further relate the galaxy-galaxy bias $b_{\mathrm{gg}}$ to dark matter clustering $\left(b_{\mathrm{gg}}^{2}=\xi_{\mathrm{gg}} / \xi_{\mathrm{DM}}\right)$ to infer the "mean" halo mass hosting O VI gas. On the latter point, we have made evaluations of $\xi_{\mathrm{DM}}$ from the code ${ }^{30}$ of Smith et al. (2003) to estimate the galaxy-galaxy bias function at $z=0.3: b_{\mathrm{gg}}=1.3 \pm 0.1$. Following the halo occupation distribution analysis of Zehavi et al. (2011) for the SDSS main survey $(z \sim 0.1)$, we relate $b_{\mathrm{gg}}$ to a characteristic halo mass $M_{h} \approx 10^{12.1 \pm 0.05} M_{\odot}$.

Figure 27 compares $\xi_{\mathrm{ag}}(r) / \xi_{\mathrm{gg}}(r)$ for the binned evaluations (left) and integrated to $4.4 h_{100}^{-1} \mathrm{Mpc}$ and we estimate $\xi_{\mathrm{ag}}(r) / \xi_{\mathrm{gg}}(r)=0.76 \pm 0.1$. This implies that O VI systems are primarily hosted by galaxies in halos with $M_{h}^{\mathrm{OVI}} \approx 10^{11} M_{\odot}$, i.e., sub- $L^{*}$ galaxies. These measurements strengthen previous assertions that O VI gas arises primarily in the surroundings of sub- $L^{*}$ galaxies based on CGM statistics (Prochaska et al. 2011b), galaxy-absorber clustering on predominantly smaller scales (Chen \& Mulchaey 2009), and linking individual galaxies to O VI absorbers (Stocke et al. 2006; Pratt et al. 2018). Future work will synthesize these

\footnotetext{
${ }^{30}$ http://cosmicpy.github.io/index.html
}

cross-correlation measurements with the statistical incidence of $\mathrm{O}$ VI to further assess the physical association of O VI to dark matter halos (e.g., Chen \& Tinker 2008).

\section{Summary}

In this paper we have reviewed the design of a photometric and spectroscopic galaxy redshift survey to support studies of the relationships between QSO absorption-line systems and galaxies, large-scale structures, and other environmental factors. We have reviewed our data handling and measurement methods as well as the content of an online public database released with this paper. Combined with absorption-line measurements from high-resolution UV CASBaH spectroscopy from HST (T. Tripp et al. 2019, in preparation), this redshift survey can be used to investigate the role of circumgalactic and intergalactic gases in galaxy evolution, and subsequent papers will exploit the data for various purposes. Importantly, both the galaxies and the absorption systems in the CASBaH database are blindly selected; no explicit preference for galaxies or absorbers of any particular type was imposed on this survey.

As an initial step in our long-term goal of investigating absorber-galaxy-environment connections, we have analyzed the clustering of $\mathrm{O}$ VI absorbers with galaxies in the CASBaH database. At small impact parameters, the CASBaH O VI systems with $\log N\left(\mathrm{O}^{+5}\right)>13.5$ and $z<0.75$ have high covering fractions that are consistent with earlier studies with different selection criteria (e.g., Prochaska et al. 2011b; Tumlinson et al. 2011; Johnson et al. 2015). This sample also exhibits a covering fraction that is larger than expected from random realizations out to very large projected distances $(\approx 8$ $\mathrm{pMpc}$ ), which indicates strong $\mathrm{O}$ VI-galaxy clustering, i.e., the gas traces the large-scale structures that comprise the cosmic web.

The clustering of O VI with galaxies is reasonably well described by a power-law cross-correlation function of the form $\xi(r)=\left(r / r_{0}\right)^{-\gamma} \quad$ with $\quad r_{0}=6.00_{-0.77}^{+1.09} h_{100}^{-1} \mathrm{Mpc} \quad$ and $\gamma=1.25 \pm 0.18$, and the bias implied by our cross-correlation analysis suggests that $\mathrm{OVI}$ absorbers are typically affiliated with dark matter halos having masses $\approx 10^{11} M_{\odot}$ at $z \sim 0.3$.

All of the spectra and photometry derived from our efforts are publicly available in a SPECDB database file that can be downloaded with that package. ${ }^{31}$ The code used to generate the figures and measurements reported here will be released on GitHub with the first set of CASBaH science papers.

J.X.P., J.K.W., J.C.H., N.L., S.L., J.B., C.N.A.W., and T.M. T. acknowledge financial support for programs HST-GO11741 and HST-GO-13846 from NASA through grants from the Space Telescope Science Institute, which is operated by the Association of Universities for Research in Astronomy, Inc., under NASA Contract NAS5-26555. J.C.H. also recognizes support from NSF grant AST-1517353, and J.K.W. appreciates support from a 2018 Alfred P. Sloan Research Fellowship. The authors would like to especially thank Michael Cooper for helpful tips in reducing the DEIMOS spectra. We also acknowledge J. Tinker for helpful discussions.

This study is also partly based on data acquired using the Large Binocular Telescope (LBT). The LBT is an international collaboration among institutions in the US, Italy, and Germany. LBT Corporation partners are the University of Arizona, on

31 https://github.com/specdb/specdb 
behalf of the Arizona university system; Istituto Nazionale do Astrofisica, Italy; LBT Beteiligungsgesellschaft, Germany, representing the Max Planck Society, the Astrophysical Institute of Postdam, and Heidelberg University; The Ohio State University, and the Research Corporation, on behalf of the University of Notre Dame, the University of Minnesota, and the University of Virginia.

The conclusions of this work are based on data collected from observatories at the summit of Maunakea. The authors wish to recognize and acknowledge the very significant cultural role and reverence that the summit of Maunakea has always had within the indigenous Hawaiian community. We are most fortunate to have the opportunity to conduct observations from this mountain.

This work has made use of data from the European Space Agency (ESA) mission Gaia (https://www.cosmos.esa.int/ gaia), processed by the Gaia Data Processing and Analysis Consortium (DPAC, https://www.cosmos.esa.int/web/gaia/ dpac/consortium). Funding for the DPAC has been provided by national institutions, in particular the institutions participating in the Gaia Multilateral Agreement. We acknowledge use of the SDSS www.sdss.org, which is funded by the Alfred P. Sloan Foundation, the U.S. Department of Energy Office of Science, the National Science Foundation, the US Department of Energy, the National Aeronautics and Space Administration, the Japanese Monbukagakusho and Participating Institutions.

The following python packages were used in our analysis: ASTROPY, LINETOOLS, PYIGM and the authors thank their developers.

\section{ORCID iDs}

J. Xavier Prochaska (ib https://orcid.org/0000-0002-7738-6875 Joseph N. Burchett (i) https://orcid.org/0000-0002-1979-2197 Todd M. Tripp (iD https://orcid.org/0000-0002-1218-640X Jessica K. Werk (10 https://orcid.org/0000-0002-0355-0134 Christopher N. A. Willmer (iD https://orcid.org/0000-00019262-9997

J. Christopher Howk (iD https://orcid.org/0000-00022591-3792

Jason Tumlinson (iD https://orcid.org/0000-0002-7982-412X Nicolas Lehner (ib https://orcid.org/0000-0001-9158-0829

\section{References}

Alam, S., Albareti, F. D., Prieto, C. A., et al. 2015, ApJS, 219, 12 Aracil, B., Tripp, T. M., Bowen, D. V., et al. 2006, MNRAS, 367, 139 Bahcall, J. N., \& Peebles, P. J. E. 1969, ApJL, 156, L7 Battisti, A. J., Calzetti, D., \& Chary, R.-R. 2016, ApJ, 818, 13 Battisti, A. J., Calzetti, D., \& Chary, R.-R. 2017, ApJ, 851, 90 Bertin, E., \& Arnouts, S. 1996, A\&AS, 117, 393

Bertin, E., Mellier, Y., Radovich, M., et al. 2002, in ASP Conf. Ser. 281, Astronomical Data Analysis Software and Systems XI, ed. D. A. Bohlender, D. Durand, \& T. H. Handley (San Francisco, CA: ASP), 228

Bond, J. R., Kofman, L., \& Pogosyan, D. 1996, Natur, 380, 603

Booth, C. M., Schaye, J., Delgado, J. D., \& Dalla Vecchia, C. 2012, MNRAS, 420, 1053

Bowen, D. V., Pettini, M., \& Blades, J. C. 2002, ApJ, 580, 169

Bruzual, G., \& Charlot, S. 2003, MNRAS, 344, 1000

Buat, V., Giovannoli, E., Heinis, S., et al. 2011, A\&A, 533, A93

Burchett, J. N., Tripp, T. M., Prochaska, J. X., et al. 2015, ApJ, 815, 91

Burchett, J. N., Tripp, T. M., Prochaska, J. X., et al. 2019, ApJL, 877, 20

Burchett, J. N., Tripp, T. M., Werk, J. K., et al. 2013, ApJL, 779, L17

Calzetti, D., Armus, L., Bohlin, R. C., et al. 2000, ApJ, 533, 682

Cantalupo, S., Arrigoni-Battaia, F., Prochaska, J. X., Hennawi, J. F., \& Madau, P. 2014, Natur, 506, 63
Cautun, M., van de Weygaert, R., \& Jones, B. J. T. 2013, MNRAS, 429, 1286 Chabrier, G. 2003, PASP, 115, 763

Chambers, K. C., Magnier, E. A., Metcalfe, N., et al. 2016, arXiv:1612.05560 Chen, H., \& Mulchaey, J. S. 2009, ApJ, 701, 1219

Chen, H.-W., Prochaska, J. X., Weiner, B. J., Mulchaey, J. S., \& Williger, G. M. 2005, ApJL, 629, L25

Chen, H.-W., \& Tinker, J. L. 2008, ApJ, 687, 745

Coil, A. L., Mendez, A. J., Eisenstein, D. J., \& Moustakas, J. 2017, ApJ, 838, 87

Croft, R. A. C., Weinberg, D. H., Bolte, M., et al. 2002, ApJ, 581, 20

Cutri, R. M., Wright, E. L., Conrow, T., et al. 2013, yCat, 2328

Dale, D. A., Helou, G., Magdis, G. E., et al. 2014, ApJ, 784, 83

Danforth, C. W., Keeney, B. A., Tilton, E. M., et al. 2016, ApJ, 817, 111

Davis, M., Efstathiou, G., Frenk, C. S., \& White, S. D. M. 1985, ApJ, 292, 371

Dawson, K. S., Schlegel, D. J., Ahn, C. P., et al. 2013, AJ, 145, 10

de Jong, J. T. A., Verdois Kleijn, G. A., Erben, T., et al. 2017, A\&A, 604, A134

Dey, A., Schlegel, D. J., Lang, D., et al. 2019, AJ, 157, 168

Eisenstein, D. J., Annis, J., Gunn, J. E., et al. 2001, AJ, 122, 2267

Faber, S. M., Phillips, A. C., Kibrick, R. I., et al. 2003, Proc. SPIE, 4841, 1657

Fabricant, D., Fata, R., Roll, J., et al. 2005, PASP, 117, 1411

Finn, C. W., Morris, S. L., Tejos, N., et al. 2016, MNRAS, 460, 590

Flewelling, H. A., Magnier, E. A., Chambers, K. C., et al. 2016, arXiv:1612. 05243

Ford, A. B., Werk, J. K., Davé, R., et al. 2016, MNRAS, 459, 1745

Gaia Collaboration, Brown, A. G. A., Vallenari, A., et al. 2016a, A\&A, 595, A2

Gaia Collaboration, Prusti, T., de Bruijne, J. H. J., et al. 2016b, A\&A, 595, A1 Ganguly, R., Lynch, R. S., Charlton, J. C., et al. 2013, MNRAS, 435, 1233

Giallongo, E., Ragazzoni, R., Grazian, A., et al. 2008, A\&A, 482, 349

Gould, A., \& Weinberg, D. H. 1996, ApJ, 468, 462

Green, J. C., Froning, C. S., Osterman, S., et al. 2012, ApJ, 744, 60

Hennawi, J. F., \& Prochaska, J. X. 2007, ApJ, 655, 735

Hewett, P. C., \& Wild, V. 2010, MNRAS, 405, 2302

Hudelot, P., Cuillandre, J.-C., Withington, K., et al. 2012, yCat, 2317

Johnson, S. D., Chen, H.-W., \& Mulchaey, J. S. 2015, MNRAS, 449, 3263

Keeney, B. A., Stocke, J. T., Pratt, C. T., et al. 2018, ApJS, 237, 11

Lan, T.-W., \& Mo, H. 2018, ApJ, 866, 36

Landy, S. D., \& Szalay, A. S. 1993, ApJ, 412, 64

Lawrence, A., Warren, S. J., Almaini, O., et al. 2007, MNRAS, 379, 1599

Lehner, N., Howk, J. C., Tripp, T. M., et al. 2013, ApJ, 770, 138

Lo Faro, B., Buat, V., Roehlly, Y., et al. 2017, MNRAS, 472, 1372

Lukić, Z., Stark, C. W., Nugent, P., et al. 2015, MNRAS, 446, 3697

McDowell, J. C., Canizares, C., Elvis, M., et al. 1995, ApJ, 450, 585

Meiring, J. D., Tripp, T. M., Werk, J. K., et al. 2013, ApJ, 767, 49

Miralda-Escudé, J., Cen, R., Ostriker, J. P., \& Rauch, M. 1996, ApJ, 471, 582

Misawa, T., Tytler, D., Iye, M., et al. 2007, AJ, 134, 1634

Morris, S. L., Weymann, R. J., Dressler, A., et al. 1993, ApJ, 419, 524

Moustakas, J., Coil, A. L., Aird, J., et al. 2013, ApJ, 767, 50

Muzahid, S., Srianand, R., Arav, N., Savage, B. D., \& Narayanan, A. 2013, MNRAS, 431, 2885

Newman, J. A., Cooper, M. C., Davis, M., et al. 2013, ApJS, 208, 5

Noll, S., Burgarella, D., Giovannoli, E., et al. 2009, A\&A, 507, 1793

Nuza, S. E., Sánchez, A. G., Prada, F., et al. 2013, MNRAS, 432, 743

Palanque-Delabrouille, N., Yèche, C., Borde, A., et al. 2013, A\&A, 559, A85

Pedregosa, F., Varoquaux, G., Gramfort, A., et al. 2011, J. Mach. Learn. Res., 12,2825

Penton, S. V., Stocke, J. T., \& Shull, J. M. 2002, ApJ, 565, 720

Pratt, C. T., Stocke, J. T., Keeney, B. A., \& Danforth, C. W. 2018, ApJ, 855,18

Prochaska, J. X., Hennawi, J. F., Lee, K.-G., et al. 2013, ApJ, 776, 136

Prochaska, J. X., Weiner, B., Chen, H.-W., Cooksey, K. L., \& Mulchaey, J. S. 2011a, ApJS, 193, 28

Prochaska, J. X., Weiner, B., Chen, H.-W., Mulchaey, J., \& Cooksey, K. 2011b, ApJ, 740, 91

Ribaudo, J., Lehner, N., \& Howk, J. C. 2011a, ApJ, 736, 42

Ribaudo, J., Lehner, N., Howk, J. C., et al. 2011b, ApJ, 743, 207

Rodrigo, C., Solano, E., \& Bayo, A. 2012, The SVO Filter Profile Service, IVOA Rep. 1015

Sand, D. J., Olszewski, E. W., Willman, B., et al. 2009, ApJ, 704, 898

Savage, B. D., Narayanan, A., Wakker, B. P., et al. 2010, ApJ, 719, 1526

Schlafly, E. F., \& Finkbeiner, D. P. 2011, ApJ, 737, 103

Simcoe, R. A., Sargent, W. L. W., \& Rauch, M. 2004, ApJ, 606, 92

Slosar, A., Iršič, V., Kirkby, D., et al. 2013, JCAP, 4, 26

Smith, R. E., Peacock, J. A., Jenkins, A., et al. 2003, MNRAS, 341, 1311

Stocke, J. T., Penton, S. V., Danforth, C. W., et al. 2006, ApJ, 641, 217 
Tejos, N., Morris, S. L., Crighton, N. H. M., et al. 2012, MNRAS, 425, 245

Tejos, N., Morris, S. L., Finn, C. W., et al. 2014, MNRAS, 437, 2017

Tempel, E., Stoica, R. S., Martínez, V. J., et al. 2014, MNRAS, 438, 3465

Tripp, T. 2013, arXiv:1303.0043

Tripp, T. M., Lu, L., \& Savage, B. D. 1998, ApJ, 508, 200

Tripp, T. M., Meiring, J. D., Prochaska, J. X., et al. 2011, Sci, 334, 952

Tripp, T. M., Sembach, K. R., Bowen, D. V., et al. 2008, ApJS, 177, 39

Tumlinson, J., Thom, C., Werk, J. K., et al. 2011, Sci, 334, 948

Verner, D. A., Barthel, P. D., \& Tytler, D. 1994, A\&AS, 108, 287
Wakker, B. P., Hernandez, A. K., French, D. M., et al. 2015, ApJ, 814, 40 Wakker, B. P., \& Savage, B. D. 2009, ApJS, 182, 378

Warren, S. J., Cross, N. J. G., Dye, S., et al. 2007, arXiv:astro-ph/0703037 Werk, J. K., Prochaska, J. X., Thom, C., et al. 2013, ApJS, 204, 17

Woodgate, B. E., Kimble, R. A., Bowers, C. W., et al. 1998, PASP, 110, 1183 Zehavi, I., Zheng, Z., Weinberg, D. H., et al. 2011, ApJ, 736, 59

Zhu, G., Ménard, B., Bizyaev, D., et al. 2014, MNRAS, 439, 3139

Zou, H., Zhou, X., Fan, X., et al. 2017a, PASP, 129, 064101

Zou, H., Zhang, T., Zhou, Z., et al. 2017b, AJ, 153, 276 